Cramer, C. J.; Truhlar, D. G. "SMx Continuum Models for Condensed Phases" in Trends and Perspectives in Modern Computational Science; Lecture Series on Computer and Computational Sciences Vol. 6; Maroulis, G., Simos, T. E., Eds.; Brill/VSP, Leiden, 2006; pp. 112-140.

\title{
SMx Continuum Models for Condensed Phases
}

\author{
Christopher J. Cramer ${ }^{1}$ and Donald G. Truhlar ${ }^{1}$ \\ Department of Chemistry and Supercomputing Institute, \\ University of Minnesota, \\ 207 Pleasant St. SE, \\ Minneapolis, MN 55455-0431, USA
}

\begin{abstract}
The SMx continuum models are designed to include condensed-phase effects in classical and quantum mechanical electronic structure calculations and can also be used for calculating geometries and vibrational frequencies in condensed phases. Originally developed for homogeneous liquid solutions, the SM $x$ models have seen substantial application to more complicated condensed phases as well, e.g., the airwater interface, soil, phospholipid membranes, and vapor pressures of crystals as well as liquids. Bulk electrostatics are accounted for via a generalized Born formalism, and other physical contributions to free energies of interaction between a solute and the surrounding condensed phase are modeled by environmentally sensitive atomic surface tensions associated with solute atoms having surface area exposed to the surrounding medium. The underlying framework of the models, including the charge models used for the electrostatics, and some of the models' most recent extensions are summarized in this report. In addition, selected applications to environmental chemistry problems are presented.

Keywords: Solvation; Polarization; Partitioning; Solubility; Thermodynamics; Vapor pressure; Electrochemistry
\end{abstract}

\section{Introduction and Underlying Physics}

Many excellent reviews of the general theory and development of continuum solvation models are available [1-13], so this contribution will not attempt to provide yet another comprehensive overview of these powerful techniques. Instead, we focus specifically on the history and present status of the SM $x$ models, which have also been reviewed [14-18], but not recently enough to include the latest developments included here. The " $x$ " in SM $x$ contains information about the model. Any number standing alone (e.g., 1, 2, 3, or 4) or preceding a decimal point (e.g., the "5" in SM5.42) indicates the generation of the model, and generations have typically been defined by a substantial change in the algorithmic approach undertaken for electrostatics, surface tensions, parameterization strategies, or some combination thereof as outlined in more detail below. Any number or numbers following a decimal point (e.g., the "42" in SM5.42) generally provide information about the charge models used to represent the solute charge distribution (this is also discussed in more detail below).

At the foundation of the SMx models is a partitioning of the free energy of transfer $\Delta G_{\mathrm{tr}}^{\mathrm{o}}$ from the gas phase to the condensed phase into two components [19]

$$
\Delta G_{\mathrm{tr}}^{\mathrm{o}}=\Delta G_{\mathrm{ENP}}^{\mathrm{o}}+G_{\mathrm{CDS}}^{\mathrm{o}}
$$

where the first term on the right-hand-side is, at the quantum mechanical level, computed as

\footnotetext{
${ }^{1}$ Corresponding authors. E-mail: cramer@umn.edu; truhlar@umn.edu.
} 


$$
\Delta G_{\mathrm{ENP}}^{\mathrm{o}}=\left\langle\Psi^{(1)}\left|H+\frac{1}{2} V\right| \Psi^{(1)}\right\rangle-\left\langle\Psi^{(0)}|H| \Psi^{(0)}\right\rangle
$$

where $\Psi^{(0)}$ and $\Psi^{(1)}$ are, respectively, the wave functions that minimize the expectation values of the gas-phase Hamiltonian $H$ and the Hamiltonian in solution; the latter adds to $H$ one half the reaction field operator $V$, which represents the field acting on the solute due to the polarization of the surrounding dielectric medium by the charge distribution of the solute. The factor of $1 / 2$ comes from linear response theory [5] and accounts for the cost of polarizing the medium. Thus, $\Delta G_{\mathrm{ENP}}^{\mathrm{o}}$ accounts for the polarization $(\mathrm{P})$ of the medium, for changes in the solute electronic $(\mathrm{E})$ structure, and, if geometry reoptimization is undertaken, for changes in the nuclear $(\mathrm{N})$ coordinates.

If the concentration of the solute is different in the liquid and vapor phases, eq. (1) needs another term to account for the associated entropy change. Our standard procedure is to use $1 \mathrm{M}$ as the concentration in both phases, use eq. (1) as written, and then change to any other standard state that may be desired, e.g., to $1 \mathrm{~atm}$ standard pressure for the vapor.

In the SMx models, the reaction field operator is represented using the generalized Born (GB) equation [6,8,19-28]. In the GB approach, the charge distribution of the solute is represented by an atomcentered distribution of monopoles (i.e., partial atomic charges) and at each atom $k$ the reaction field is defined as

$$
V_{k}=\left(1-\frac{1}{\varepsilon}\right) \sum_{k^{\prime}}^{\mathrm{atoms}} q_{k^{\prime}} \gamma_{k k^{\prime}}
$$

where $\varepsilon$ is the dielectric constant of the medium, $q_{k}$ is a partial atomic charge, and $\gamma_{k k^{\prime}}$ is an effective Coulomb integral first suggested by Still et al. [25]

$$
\gamma_{k k^{\prime}}=\left(r_{k k^{\prime}}^{2}+\alpha_{k} \alpha_{k^{\prime}} e^{-r_{k k^{\prime}}^{2} / d_{k k^{\prime}} \alpha_{k} \alpha_{k^{\prime}}}\right)^{-1 / 2}
$$

where $r_{k k^{\prime}}$ is the interatomic distance, $\alpha$ is an effective atomic Born radius, $d_{k k^{\prime}}$ is a parameter chosen by Still et al. [25] to be 4 and by us to be either 4 or some value rather close to 4 and $\alpha$ is an effective Born radius.

The effective Born radius may be calculated in a number of different ways [19,25,28-36] which will not be reviewed in detail here. Conceptually, it is the radius that a spherical monatomic ion - having a charge equal to the partial charge of the atom in the solute-would need to have in order to have the same solvation free energy in the medium as does the solute atom embedded in the full, otherwise uncharged solute (the volume of which, by displacing the dielectric medium, descreens the particular atom in question). The free energy of solvation of the atom embedded in the solute is computed by solving the Poisson equation for the charged species in the dielectric medium. The necessary integration for this solution is accomplished either numerically or by using an analytical quadrature approach, and one aspect particularly worthy of attention here is that some lower limit for the integration must be chosen; in SMx models this limit for a monatomic ion is referred to as the intrinsic or atomic Coulomb radius $\rho_{k}$ and the determination of robust atomic $\rho$ values is a key aspect in parameterization.

The second term on the r.h.s. of eq. (1) refers to the free energy of cavitation (C), dispersion (D), and changes in the otherwise homogeneous solvent structure (S) induced by the solute. The SM $x$ models assume that these various free energies may be partitioned into atomic contributions, and that each atom's contribution will depend on its atomic number, its intramolecular environment, and the extent to which it is exposed to the surrounding medium. Thus, we compute

$$
G_{\mathrm{CDS}}^{\mathrm{o}}=\sum_{k}^{\text {atoms }} \sigma_{k}(\mathbf{Q}) A_{k}
$$


where $A_{k}$ is the solvent accessible surface area $[28,37,38]$ of atom $k$ and $\sigma_{k}$ has units of surface tension and is not necessarily a simple constant for all atoms of a given atomic number but instead a function of the nuclear coordinates $\mathbf{Q}$ and, in SM2 and SM3, on functions of the electronic density matrix as well.

We next proceed to detail how the SM $x$ models have evolved within the general framework described by eqs. (1)-(5). We pay special attention to pointing out the details that distinguish one SMx model from another.

\section{A Brief History of the SMx Models for Homogeneous Liquid Solutions}

2.1. SMx models for semiempirical Hamiltonians. The first SMx models to be reported were SM1 and SM1 a [19]. These two models were the first quantum mechanical continuum solvation models to be parameterized against an extensive set of experimental aqueous free energies of solvation (141 neutral compounds and 27 ions; most prior models had tended to focus on specific components of the solvation free energy that are not physical observables, or had focused on only one or a very small number of free energies of solvation for related molecules). Parameterization against increasingly large experimental data sets has been a hallmark of all SMx model development.

Both SM1 and SM1a were based on the semiempirical Austin Model 1 (AM1) Hamiltonian [39]. In these first generation models, the partial atomic charges used to define the reaction field were taken calculated from the electronic density matrix by the population analysis of Mulliken (which reduces to the description of Coulson within the context of a zero diatomic overlap model like AM1) [40]. Thus, Fock matrix elements in the self-consistent reaction field (SCRF) process were defined as

$$
F_{\mu v}^{(1)}=F_{\mu v}^{(0)}+\delta_{\mu v}\left(1-\frac{1}{\varepsilon}\right) \sum_{k^{\prime}} \sum_{v \in k^{\prime}}\left(Z_{k^{\prime}}-P_{v v}\right) \gamma_{k k^{\prime}}
$$

where $\mathbf{F}$ is the Fock matrix indexed over basis functions $\mu$ and $v, \delta$ is the Kronecker delta, $Z$ is the AM1 valence nuclear charge, and $\mathbf{P}$ is the density matrix. The Coulomb integral $\gamma_{k k}{ }^{\prime}$ was defined as in eq. (4) except for $\mathrm{OO}$ and $\mathrm{NH}$ atom pairs, for which an additional term was added to effectively increase the screening between these two charges.

Another feature of SM1 and SM1a was that the atomic Coulomb radii were made to vary as a function of partial atomic charge. In particular, we used

$$
\rho_{k}=\rho_{k}^{(0)}-\rho_{k}^{(1)}\left[\frac{1}{\pi} \tan ^{-1}\left(\frac{q_{k}+q_{k}^{(0)}}{q^{(1)}}\right)+\frac{1}{2}\right]
$$

where $\rho_{k}^{(0)}, \rho_{k}^{(1)}$, and $q_{k}^{(0)}$ are atom-specific parameters, and $q^{(1)}$ was a universal parameter set to 0.1 . The idea of charge-dependent radii has some intuitive conceptual appeal, and such a treatment persisted through SM4. However, the approach was abandoned in favor of constant Coulomb radii beginning with SM5-type models. There were several reasons for this choice. First, atomic partial charges tend to cluster in discrete, narrow regions, making it difficult to develop switching functions between those regions that are not fairly arbitrary. Unfortunately, while such arbitrariness may not affect typical stable molecules, there may be much more significant effects on transition-state structures, where atoms may be passing between standard hybridizations and charges, so the consequences for reaction dynamics may be large. In addition, because SM1 and SM1 a were designed for use at the semiempirical level, it was not terribly expensive to carry out numerical geometry optimizations. However, in order to compute analytic derivatives it is quite inconvenient to have the Coulomb radii, and thus the effective Born radii, depend on density matrix elements in a particularly complicated way. In unpublished work preceding the development of the SM6 solvation model, we (C. Kelly, C. J. Cramer, and D. G. Truhlar) revisited the issue of whether more accurate models could be obtained by letting the intrinsic Coulomb 
radii depend linearly or quadratically on particular atomic charges, but the gain in accuracy was insignificant.

With respect to $G_{\mathrm{CDS}}^{\mathrm{o}}$, SM1 used the simplest possible approach for defining $\sigma_{k}$ in eq. (6): the surface tension depended only on the atomic number of atom $k$. SM1 a, on the other hand, parameterized surface tensions based on atom "types", much as in molecular mechanics, and was designed to be used only for neutral molecules. Thus, the user would assign a type for each atom (e.g., ether oxygen or nitrile nitrogen) and the surface tension would be chosen accordingly. Over the parameterization training set of neutrals, SM1 and SM1 a exhibited root-mean-square (rms) errors of 1.52 and 0.78 $\mathrm{kcal} / \mathrm{mol}$, respectively. Over the ionic training set, the rms error for SM1 was $4.4 \mathrm{kcal} / \mathrm{mol}$. The models were defined for molecules containing $\mathrm{H}, \mathrm{C}, \mathrm{N}, \mathrm{O}, \mathrm{F}, \mathrm{S}, \mathrm{Cl}, \mathrm{Br}$, and $\mathrm{I}$.

The next two SMx models to be reported, SM2 [26,41] and SM3 [26,42], were also aqueous solvation models based on semiempirical Hamiltonians. SM2 was designed for use with AM1 and SM3 for use with the Parameterized Model 3 (PM3) of Stewart [43]. The key difference between SM2 and SM3 and their precursor SM1 is in the determination of $G_{\mathrm{CDS}}^{\mathrm{o}}$. In essence, recognizing the significant improvement SM1a offered over SM1, some of the heuristic principles by which a chemist assigns atom types were encoded into algorithms that assigned surface tension not only on the basis of atomic number, but also based on the number of attached hydrogen atoms, which were taken to have zero atomic radius in the computation of $G_{\mathrm{CDS}}^{\mathrm{o}}$. In essence, then, SM2 and SM3 are united-atom models where heavy-atom properties are modified based on the hydrogen atoms attached to them.

Heavy atom surface tensions were defined as

$$
\sigma_{k}=\sigma_{k}^{(0)}+\sigma_{k}^{(1)}\left[f\left(B_{k \mathrm{H}}\right)+g\left(B_{k \mathrm{H}}\right)\right]
$$

where $\sigma_{k}^{(0)}$ and $\sigma_{k}^{(1)}$ are atom-specific parameters and $f$ and $g$ are functions of the bond order matrix $\mathbf{B}$ $[44,45]$, the first being defined as

$$
f\left(B_{k \mathrm{H}}\right)=\tan ^{-1}\left(\sqrt{3} B_{k \mathrm{H}}\right)
$$

and the second being a function targeted only to oxygen and nitrogen and designed to correct for otherwise systematic errors in $\mathrm{H}_{2} \mathrm{O}, \mathrm{NH}_{3}$, and primary oxonium and ammonium ions. Note that the bond order matrix is a function of the electronic density matrix [45].

Eq. (8) permits substantial distinction between different functional groups, and its adoption caused SM2 and SM3 to be much more accurate for the prediction of the free energies of solvation for hydrophobic species. The parameterization set for SM2 and SM3 was also extended by 9 molecules compared to SM1, which permitted P to be added to the list of allowed atoms in solute molecules, although parameterizations for P were not entirely reliable until SM5.42. Over the neutral set, SM2 and SM3 had rms errors of 0.9 and $1.3 \mathrm{kcal} / \mathrm{mol}$, respectively, and over the ionic set these errors were 3.9 and $5.6 \mathrm{kcal} / \mathrm{mol}$, respectively. The larger errors exhibited by SM3 are associated in part with the now well known poor quality of PM3 partial charges on $\mathrm{N}$ atoms [26].

Because of their ready availability in the free code AMSOL [46] and the commercial code SPARTAN [47], the SM2 and SM3 models saw substantial use after their introduction [48-92]. Subsequent to their original development, improvements were made in the numerical stability of certain aspects of the calculation (e.g., the quadrature scheme used for integration in determining effective Born radii, the analytical approach used for the computation of molecular surface area, and the updating scheme used in construction of the Fock matrix including the reaction field operator), and more robust versions of SM2 and SM3 were produced and called SM2.1 and SM3.1, respectively [28]; results from these models were practically unchanged compared to their precursors. The numerical improvements introduced into SM2.1 and SM3.1 have been incorporated into all subsequent SM $x$ models as well. SM2, SM3, SM2.1, and SM3.1 are no longer recommended since several models in the SM5 series 
discussed below are parameterized more broadly and more accurately for AM1 and PM3 as well as the equally inexpensive Modified Neglect of Diatomic differential Overlap (MNDO) model.

The SM4 models were the first to introduce a significant change in the calculation of $\Delta G_{\mathrm{ENP}}^{\mathrm{o}}$. These models continued to be founded on the semiempirical Hamiltonians AM1 and PM3, but instead of using Mulliken partial atomic charges in the reaction field operator, they made use of Charge Model 1 (CM1) to compute Class IV partial atomic charges [93]. The CM1 partial atomic charge is defined as

$$
q_{k}^{\mathrm{CM} 1}=q_{k}^{(0)}+B_{k} \Delta q_{k}-\sum_{k^{\prime} \neq k} B_{k k^{\prime}} \Delta q_{k^{\prime}}
$$

where $q_{k}^{(0)}$ is a reference charge (e.g., a semiempirical Mulliken charge), $B_{k k^{\prime}}$ is the covalent bond order (also called bond index) [45] between atoms $k$ and $k^{\prime}, B_{k}$ is the sum of all bond orders from atom $k$ to all other atoms, and $\Delta q_{k}$ is defined as

$$
\Delta q_{k}=c_{k} q_{k}^{(0)}+d_{k}
$$

where $c$ and $d$ are an empirically optimized scaling parameter and offset, respectively, for atom $k$. The form of eq. 10 ensures that total molecular charge is conserved in the CM1 mapping procedure. The parameter vectors $\mathbf{c}$ and $\mathbf{d}$ for all atoms are optimized so that molecular dipole moments computed from the distributed partial atomic charges best fit an experimental data set for neutral molecules, and partial atomic charges computed from electrostatic potentials for select charged molecules [93]. CM1 charges are much more accurate then semiempirical Mulliken charges for the computation of molecular electrical moments, leading to a more physical partition of the total solvation free energy into ENP and CDS components when they are used in the GB procedure (note that by the nature of the parameterization of the surface tension terms, errors in the ENP component are perforce absorbed - as well as possible-into the CDS component).

The first SM4 model to be reported was not for water as solvent, but instead for $n$-hexadecane [94] (a solvent for which many data are available because it can be used as a stationary phase in capillary gas chromatography). For the hexadecane model, another new feature was also introduced into the SM4 model, namely, the use of different solvent radii to compute solvent accessible surface areas associated with different physical components of the CDS term. In particular, a CD area and a CS area were identified separately and the full CDS term was computed as

$$
G_{\mathrm{CDS}}^{\mathrm{o}}=\sigma^{\mathrm{CS}} \sum_{k} A_{k}^{\mathrm{CS}}+\sum_{k} \sigma_{k}^{\mathrm{CD}}(\mathbf{Q}) A_{k}^{\mathrm{CD}}
$$

where $\sigma^{\mathrm{CS}}$ is an empirically optimized molecular surface tension associated with a large solvent probe radius to generate the molecular surface area $A^{\mathrm{CS}}$ and $\sigma^{\mathrm{CD}}$ is an atom-specific surface tension that may either be an empirically optimized constant, or a function of empirical constants and bond orders between atom $k$ and other atoms. In the latter case, the spirit of the approach taken in SM2 and SM3 was preserved, but the united atom model was abandoned in favor of a situation in which each $\mathrm{H}$ atom had a surface tension influenced by the atom attached to it, rather than vice versa. Atomic CD surface areas were computed with smaller probe radii, under the assumption that dispersion interactions occur over a much shorter range than do changes in the solvent structure when a solute is introduced.

Preliminary AM1-SM4 and PM3-SM4 models for $n$-hexadecane were parameterized against data for 153 neutral molecules. However, their parameters and performances were so similar to one another (because the CM1 charge mapping results in charge distributions that are effectively independent of the underlying Hamiltonian) that a single set of parameters was optimized for use with either Hamiltonian and taken to define SM4-hexadecane. The rms error of this model over the 306 data derived from combining the AM1 and PM3 calculations was $0.41 \mathrm{kcal} / \mathrm{mol}$ (for comparison, the dispersion in the reference data was $2.05 \mathrm{kcal} / \mathrm{mol})$. 
Subsequent work extended the SM4 model to other alkanes [95]. A data set of 350 solute/solvent combinations, where the solvents spanned 16 different alkanes, was constructed and added to the $n$-hexadecane data set. Since the alkanes would be expected to behave very similarly with one another when it comes to dispersion interactions with a solute, and since the dielectric constants are known, it was assumed that the only model parameter that one might assume to vary as a function of solvent would be $\sigma^{\mathrm{CS}}$ in eq. (12). The free energy of cavitation/structural rearrangement was assumed to be proportional to the macroscopic surface tension $\gamma$ of the solvent and empirical optimization established that for all alkanes

$$
\sigma^{\mathrm{CS}}=0.03332 \gamma+15.95 \mathrm{cal} / \mathrm{mol} \bullet \AA
$$

provided an excellent fit to all of the experimental data (eq. (13) was constrained to reproduce SM4hexadecane exactly). Values of $\sigma^{\mathrm{CS}}$ computed from eq. (13) differed from values optimized on a solvent-by-solvent basis by no more than $0.02 \mathrm{cal} / \mathrm{mol} \bullet \AA$. Again, a single set of parameters was found to be equally applicable to both the AM1 and PM3 Hamiltonians, and the rms error over all data was $0.45 \mathrm{kcal} / \mathrm{mol}$.

An aqueous SM4 model for water was never developed. In two cases, preliminary work was reported for solutes containing $\mathrm{C}, \mathrm{H}$, and $\mathrm{O}$ atoms. However, those cases were not general parameterizations, but were instead restricted to certain classes of compounds for use in modeling aqueous solvation effects on the Claisen rearrangement [14] and sugar conformational analysis [96]. Careful analysis of work accomplished up to that point indicated that the dependence of surface tensions and Coulomb radii on bond orders and partial atomic charges led to instabilities associated with the difficulty of properly including these terms in Fock matrix updates as part of the SCRF equations. A decision was taken to alleviate this problem by eliminating such dependencies, and the resulting new protocol was referred to as SM5.

In practice, the term "SM5" does not refer to any specific model, but instead represents a general functional form wherein (i) all Coulomb radii are taken to be independent of partial atomic charge, (ii) Eq. (4) holds for all pairwise atomic combinations without exception, (iii) the van der Waals radii of Bondi [97] together with a solvent probe radius are used for the calculation of solvent-accessible surface area for use in computing $G_{\mathrm{CDS}}^{\mathrm{o}}$, and (iv) all atomic surface tensions are taken to depend only on atomic number and local solute geometry, not on bond orders. Except for $\mathrm{H}$, all Coulomb radii are taken to be constant. For $\mathrm{H}$ atoms bonded to $\mathrm{N}$ and $\mathrm{O}$, a somewhat smaller radius is assigned based on a geometric function that identifies such bonding. The dependence of atomic surface tensions on molecular geometry is, ultimately, simply a more computationally convenient and unambiguous way of encoding the heuristic rules so successfully used for assigning atomic types in the SM1a model, but with the advantage that the rules are smooth and differentiable functions of atomic coordinates, making quantum calculations on unusual species and reaction coordinates entirely feasible.

With respect to specific models, SM5 models are always identified by additional characters in their names. When CM1 Class IV charges are used in the computation of $\Delta G_{\mathrm{ENP}}^{\mathrm{o}}$, and surface tensions are optimized for the corresponding $G_{\mathrm{CDS}}^{\mathrm{o}}$, the model is referred to as SM5.4. When Class II semiempirical Mulliken charges and the resulting associated surface tensions are used, the model is designated SM5.2. When $\Delta G_{\mathrm{ENP}}^{\mathrm{o}}$ is ignored completely, and the total solvation free energy is assumed to be computable entirely from eq. (5), the model is named SM5.0. In addition, during the course of the development of all of the SMx models, it was observed that reoptimization of geometries typically contributes at most $5 \%$ to a total solvation free energy. As a result, computation of SCRF solvation free energies at gasphase geometries is an efficient alternative, and when geometries are restricted to the gas phase, the model may be identified by appending an "R" to the model name, e.g., SM5.0R. Later work at HartreeFock (HF) and density functional theory (DFT) levels, however, has tended to adopt instead the usual "double-slash" notation (where levels for energies are specified prior to the double slash and geometries are specified afterwards, e.g., $\mathrm{SM} x / \mathrm{HF} / 6-31 \mathrm{G} / / \mathrm{HF} / 6-31 \mathrm{G})$.

Another suffix that may be added to the SM5 notation is PD, for "pairwise descreening" [30,98]. At semiempirical levels, solution of the SCF equations is sufficiently fast that the computation of effective Born radii can become the slowest step in the SCRF calculation. Pairwise descreening approximates 
the difficult integral involved in computing the atomic solvation free energy with a parametrically scaled combination of pairwise, analytic integrals between the atom in question and all other atoms. This scheme speeds up the calculation considerably, and indeed the PD approach ${ }^{2}$ has been widely employed in classical GB schemes (where atomic partial charges are force field parameters) in order to take advantage of its greater speed, especially for calculations on biopolymers $[99,100]$. At the HF and DFT levels the computation of effective Born radii no longer comprises a significant fraction of the effort, so most modern SMx calculations do not adopt the PD approach, although it remains an efficient scheme for large- or multi-scale molecular dynamics calculations. The original report [30] of the PD approach reoptimized surface tensions for SM2.1 within the context of PD descreening and named a preliminary version of this method SM2.2; subsequent work completed this parameterization and adopted the PD suffix [98].

Given that overall framework, the first SM5 model to be published [101] described the SM5.4A and SM5.4P aqueous solvation models (designed for use with AM1 and PM3, respectively); their rms errors over an expanded test set of 215 aqueous solvation free energies of neutral molecules were 0.72 and $0.62 \mathrm{kcal} / \mathrm{mol}$, respectively. Over 34 ions their rms errors were 5.7 and $5.4 \mathrm{kcal} / \mathrm{mol}$, respectively. A key advance accomplished at the SM5 level was the extension of the model to organic solvents (briefly called OSM5.4 to emphasize its "organic" nature, although this nomenclature is no longer used) $[102,103]$. This extension was done in a general way such that the model was (and still is) referred to as being "universal". As in all SM5 models, the ENP term is computed using the normal GB formalism and the appropriate dielectric constant for the solvent. The CDS term, on the other hand, is computed from eq. (12) using solvent probes of 1.7 and $3.4 \AA$ for the CD and CS surface areas, respectively, but the $\mathrm{CD}$ and $\mathrm{CS}$ surface tensions themselves are made to be linear functions of macroscopic solvent descriptors. In particular

$$
\sigma_{k}^{\mathrm{CD}}=\sum_{\lambda=n, \alpha, \beta} \sigma_{k}^{\mathrm{CD}, \lambda} \lambda
$$

where all parameters implicit in each $\sigma_{k}^{\mathrm{CD}, \lambda}$ are empirically optimized over a data set comprising free energies of solvation into multiple organic solvents, $n$ is the solvent index of refraction, $\alpha$ is Abraham's hydrogen bonding acidity $\sum \alpha_{2}^{\mathrm{H}}$ [104], and $\beta$ is Abraham's hydrogen bonding basicity $\sum \beta_{2}^{\mathrm{H}}[104]$. For the molecular surface tension, we took

$$
\sigma^{\mathrm{CS}}=\sum_{\lambda=n, \gamma} \sigma^{\mathrm{CS}, \lambda} \lambda
$$

where all symbols have been described above.

Over a data set of 1786 free energies of solvation for 206 different solutes in 90 different organic solvents, the SM5.4/AM1 and SM5.4/PM3 models achieved mean unsigned errors of under 0.5 $\mathrm{kcal} / \mathrm{mol}$ after parameterization. For chloroform [105] and benzene and toluene [103], refined models were described where solvation free energies for solutes into these solvents were heavily overweighted in the optimization of the parameters implicit in equations (14) and (15). Systematic errors in these solvents were eliminated in this fashion, although the magnitude of these errors was less than 1 $\mathrm{kcal} / \mathrm{mol}$ in terms of mean unsigned error.

Aqueous solvation models using the SM5 functional forms for surface tensions but combining these with $\Delta G_{\mathrm{ENP}}^{\mathrm{o}}$ computed from Class II Mulliken charges in combination with pairwise descreening were reported for the AM1 and PM3 Hamiltonians and referred to as SM5.2PD models [98]. Aqueous pairwise descreening models SM5.4PD were also developed [98]. All of the models achieved similar overall accuracies, but the partitioning between ENP and CDS components is presumably more realistic with Class IV charges and without adopting the PD approximation. Subsequently, SM5.2

\footnotetext{
${ }^{2}$ This is sometimes referred to in the literature as the HCT approximation, for Hawkins, Cramer, and Truhlar.
} 
organic models were developed for AM1, PM3, MNDO, and MNDO/d [106-109], again achieving accuracy similar to SM5.4 analogs at reduced cost (primarily because of simpler coding) but with some loss of physicality.

At the extreme end of sacrificing physicality for speed, it was discovered that eliminating the computation of $\Delta G_{\mathrm{ENP}}^{\mathrm{o}}$ altogether and computing the full aqueous free energy of solvation using only SM5 surface tension functionals led to a model, SM5.0, that, was essentially as accurate as any of the other SM5 aqueous models for neutral solutes [110]. The SM5.0 model does not require any electronic structure calculations, so it is extraordinarily fast, although molecular geometries must be chosen in some fashion, of course. The SM5.0 model was subsequently extended in a universal way to the organic parameterization set [111] and a freely distributed code, OMNISOL [112], was created incorporating these models. Another model, SM5.05, was also described [110] that was designed to permit an extension of aqueous SM5.0 to include the charged groups found in protein side chains at neutral $\mathrm{pH}$, e.g., imidazolium ions, carboxylate anions, primary ammonium cations, and guanidinium ions.

Finally, SM5 surface tension functional forms and parameters have been optimized for use with $\Delta G_{\mathrm{ENP}}^{\mathrm{o}}$ values computed by means other than the GB formalism. In particular, SM5C [113] uses the SM5 surface tension functional form but computes $\Delta G_{\mathrm{ENP}}^{\mathrm{o}}$ at the AM1, PM3, MNDO, and MNDO/d level from the conductor-like screening model (COSMO) of Klamt and co-workers [114]. A key difference between the GB and COSMO approaches is that the latter uses the full electron density to represent the solute charge distribution instead of atom-centered point charges, and there may be instances where this more complete representation yields a more physical partitioning between electrostatic and non-electrostatic components of the full free energy of solvation. In practice, however, the accuracy of the SM5C model over the full SM5 training set is about the same as that observed for any of the other SM5-type models.

2.2. SMx models for ab initio Hartree-Fock theory and density functional theory. All of the quantum mechanical model development described for the SMx models thus far relied on underlying semiempirical QM levels of theory. Early efforts to extend the models to the HF and DFT levels made it clear, however, that the CM1 mapping scheme was not particularly well suited to these more complete levels of theory: Mulliken charges and bond orders show strong basis-set dependence and take on very unphysical values as basis sets become more saturated. In order to create a better charge mapping scheme for use at the HF and DFT levels, Charge Model 2 (CM2) was developed [115,116]. The CM2 mapping may be regarded as a more flexible and simpler extension of the CM1 model with the simultaneous adoption of pairwise specific parameters describing charge transfer between bonded atoms. The CM2 partial atomic charge is defined as

$$
q_{k}^{\mathrm{CM} 2}=q_{k}^{(0)}+\sum_{k^{\prime} \neq k} B_{k k^{\prime}}\left(D_{k k^{\prime}}+C_{k k^{\prime}} B_{k k^{\prime}}\right)
$$

where the reference charge $q_{k}^{(0)}$ is now taken to be a Löwdin charge [117] (to reduce basis-set dependence), $B_{k k^{\prime}}$ is the Mayer bond order between atoms $k$ and $k^{\prime}[118,119]$, and $C_{k k^{\prime}}$ and $D_{k k^{\prime}}$ are empirically optimized parameters that change sign upon reversal of $k$ and $k^{\prime}$. Over a database of 211 polar molecules, CM2 models specific to level of theory/basis set combinations predict dipole moments with rms errors on the order of $0.2 \mathrm{D}$.

Subsequent SMx model development using CM2 charges led to the creation of SM5.42 models, where the "4" again denotes Class IV charges and the final " 2 " indicates that the Class IV charges come from CM2. At the HF level, the first SM5.42 model [120] was an aqueous model parameterized for the MIDI! basis set $[121,122]$ based on gas-phase HF/MIDI! geometries. In short order, aqueous and organic SM5.42 models were described for a large number of additional HF and DFT levels /basis set

combinations $[123,124]$. For organic solvents, eq. (15) was modified so that the summation runs over 4 solvent descriptors: the macroscopic surface tension $(\gamma)$ the square of Abraham's hydrogen bonding 
basicity $\left(\beta^{2}\right)$ the square of the fraction of solvent heavy atoms that are either $\mathrm{F}, \mathrm{Cl}$, or $\mathrm{Br}\left(\phi^{2}\right)$, and the square of the fraction of solvent heavy atoms that are aromatic carbon atoms $\left(\psi^{2}\right)$. Over all solvents and solutes, mean unsigned errors in neutral solvation free energies were found typically to be on the order of $0.5 \mathrm{kcal} / \mathrm{mol}$ and the errors for ions in aqueous solution were about an order of magnitude larger. Solutes containing Si were added to the training set so that parameters for this atom could be determined [125]; predictive accuracies for the training set molecules containing Si are similar to all others.

In addition to $a b$ initio levels of theory, a CM2 model was developed [126] for the INDO/S semiempirical Hamiltonian $[127,128]$ based on a training set containing both ground- and excited-state charge distributions. An SM5.42/INDO model for ground-state molecules [129] was built upon this CM2 model, as was a two-time-scale electrostatics-only vertical excitation model (VEM4.2) designed to predict solvatochromism in UV absorption spectra [130]. This model, augmented by dispersion and hydrogen bonding terms, was used very successfully to predict the solvatochromic shifts of acetone in nine solvents.

During the course of this development effort, analytic derivatives were derived for SM5.42 solvation free energies [131], permitting efficient optimization of molecular geometries in solution. As of this date, analytic second derivatives have not yet been derived, although vibrational frequencies may be determined from numerical differentiation of the analytic first derivatives.

The SM5.42 models may be regarded as being reasonably mature, and they continue to be useful for modern research. Nevertheless, they are not the most modern members of the SM $x$ family. In order to improve further upon the electrostatics in the GB treatment, a new Charge Model 3 [132-135] was developed that did not differ from CM2 in functional form, but was parameterized over a much more diverse test set roughly double the size of that used for CM2. In addition, a charge renormalization scheme was adopted for use with basis sets containing diffuse functions since with such basis sets Löwdin charges and Mayer bond orders were otherwise found to be less reliable [136].

SM5-type models making use of CM3 charges are referred to as SM5.43 models [137,138]. Aqueous and organic models with analytic gradients are available for the HF and B3LYP [139-142] levels with the $6-31 \mathrm{G}(\mathrm{d})$ basis set $[143]$. They are also available for the MPW $X$ density functional, which corresponds to mixing $X \%$ of Hartree-Fock nonlocal exchange with $(100-X) \%$ of local $m \mathrm{PW}$ exchange and $100 \%$ of PW91 density functional correlation, with $X$ taking on any value from 0 to $100 \%$ [144146], with any of the MIDI!, 6-31G(d), 6-31+G(d), or 6-31+G(d,p) basis sets. The models are coded in the freely distributed software packages GAMESSPLUs [147], HONDOPLUS [148], and SM $x$ GAUSS [149], and their inclusion in other codes is ongoing (updates may be found on the comp.chem.umn.edu website). Comparisons of select SM5.43 models to some of the most recent versions of the polarized continuum model (PCM; [150-155]), which is also widely used for continuum solvation calculations, are presented in Tables 1 and 2 for aqueous and organic solvents, respectively. The superior performance of the SM5.43 models is noteworthy, particularly for organic solvents.

The most recently developed SM $x$ model is SM6, which is presently available only for water [156]. Charge model 4 (CM4) [156] was developed as part of the SM6 parameterization. The primary difference between CM3 and CM4 is that the former was judged, after detailed analysis, to sometimes predict $\mathrm{C}-\mathrm{H}$ bonds to be too polar. The CM4 parameterization therefore began by optimizing the $C_{\mathrm{CH}}$ and $D_{\mathrm{CH}}$ parameters so as best to reproduce the Optimized Potentials for Liquid Simulations (OPLS;

[157]) partial atomic charges for 19 hydrocarbons. ${ }^{3}$ Subsequent to that, all other parameters were optimized in the same fashion as for CM3. SM6 also has a neutral training set with some additional organic functionality not considered for SM5.43 and previous models. More importantly, however,

\footnotetext{
${ }^{3}$ Although the OPLS partial charges are optimized for liquid phases, where dielectric screening makes bonds more polar than in the gas phase, the difference is expected to be small for $\mathrm{C}-\mathrm{H}$ bonds, and we elected to use these charges as target values for gas-phase calculations.
} 
Table 1: Mean unsigned error of the aqueous free energy of solvation $(\mathrm{kcal} / \mathrm{mol})$ of various solute classes calculated by various continuum solvation models using $\mathrm{HF} / 6-31 \mathrm{G}(\mathrm{d})$

\begin{tabular}{|c|c|c|c|c|c|c|}
\hline solute class & no. data & SM5.42 & C-PCM ${ }^{a}$ & D-PCM ${ }^{a}$ & IEF-PCM $^{a}$ & SM5.43 \\
\hline neutral $\mathrm{H}, \mathrm{C}, \mathrm{N}, \mathrm{O}, \mathrm{F}^{b}$ & 171 & 0.57 & 0.79 & 1.21 & 0.76 & 0.51 \\
\hline $\mathrm{Cl}, \mathrm{Br}, \mathrm{S}$, and $\mathrm{P}$ neutrals $\mathrm{s}^{c}$ & 86 & 0.49 & 1.64 & 1.69 & 1.64 & 0.48 \\
\hline all neutrals & 257 & 0.54 & 1.07 & 1.37 & 1.06 & 0.50 \\
\hline $\mathrm{H}, \mathrm{C}, \mathrm{N}, \mathrm{O}, \mathrm{F}$ ions $^{b}$ & 32 & 5.20 & 7.66 & 9.18 & 7.63 & 4.91 \\
\hline $\mathrm{Cl}, \mathrm{Br}, \mathrm{P}, \mathrm{S}$ ions ${ }^{c}$ & 15 & 4.03 & 2.16 & 13.12 & 2.18 & 4.10 \\
\hline all ions & 47 & 4.83 & 5.90 & 10.44 & 5.89 & 4.65 \\
\hline
\end{tabular}

${ }^{a}$ As coded in Gaussian 03

${ }^{b}$ Solutes containing at most the five listed elements

${ }^{c}$ Solutes containing at least one of these elements plus, in most cases, elements from the previous row

Table 2: Mean unsigned error of the free energy of solvation $(\mathrm{kcal} / \mathrm{mol})$ of solutes in the indicated solvent calculated by various continuum solvation models with the $6-31 \mathrm{G}(\mathrm{d})$ basis set

\begin{tabular}{|c|c|c|c|c|c|c|}
\hline \multirow[b]{2}{*}{ solute class } & \multirow[b]{2}{*}{ no. data ${ }^{a}$} & \multicolumn{4}{|c|}{ B3LYP } & \multirow{2}{*}{$\frac{m \mathrm{PW} 1 \mathrm{PW} 91}{\mathrm{SM} 5.43}$} \\
\hline & & C-PCM ${ }^{b}$ & D-PCM ${ }^{c}$ & IEF-PCM $^{c}$ & SM5.43 & \\
\hline acetonitrile & 7 & 5.22 & 5.13 & 5.22 & 0.37 & 0.41 \\
\hline aniline & 9 & 7.55 & 7.54 & 7.73 & 0.50 & 0.50 \\
\hline benzene & 68 & 4.81 & 5.09 & 5.15 & 0.62 & 0.63 \\
\hline carbon tetrachloride & 72 & 4.53 & 4.76 & 4.80 & 0.48 & 0.49 \\
\hline chlorobenzene & 37 & 3.90 & 4.01 & 4.12 & 0.51 & 0.55 \\
\hline chloroform & 96 & 4.56 & 4.75 & 4.82 & 0.53 & 0.55 \\
\hline cyclohexane & 83 & 2.30 & 2.52 & 2.53 & 0.40 & 0.41 \\
\hline dichloroethane & 38 & 3.85 & 3.94 & 4.05 & 0.43 & 0.44 \\
\hline diethyl ether & 62 & 3.26 & 3.49 & 3.68 & 0.62 & 0.63 \\
\hline dimethyl sulfoxide & 7 & 3.42 & 3.31 & 3.43 & 0.57 & 0.61 \\
\hline ethanol & 8 & 2.17 & 2.45 & 1.87 & 1.30 & 1.35 \\
\hline heptane & 60 & 2.21 & 2.46 & 2.49 & 0.33 & 0.34 \\
\hline methylene chloride & 11 & 2.01 & 3.16 & 3.26 & 0.45 & 0.47 \\
\hline nitromethane & 7 & 2.83 & 2.64 & 2.84 & 0.67 & 0.71 \\
\hline tetrahydrofuran & 7 & 3.12 & 3.10 & 3.20 & 0.32 & 0.33 \\
\hline toluene & 49 & 3.43 & 3.67 & 3.75 & 0.40 & 0.41 \\
\hline 16 above solvents & 621 & 3.68 & 3.89 & 3.96 & 0.49 & 0.51 \\
\hline all other 74 solvents & 1359 & n.a. ${ }^{d}$ & n.a. ${ }^{d}$ & n.a. ${ }^{d}$ & 0.50 & 0.52 \\
\hline self-solvation energies ${ }^{e}$ & $76(16)^{f}$ & $(3.94)$ & $(4.14)$ & $(4.20)$ & $0.50(0.49)$ & $0.53(0.51)$ \\
\hline
\end{tabular}

${ }^{a}$ Number of experimental data in this solvent

${ }^{b}$ As coded in Gaussian 98

${ }^{c}$ As coded in Gaussian 03

$d_{\text {not available }}$

${ }^{e}$ Standard-state free energy of solvation of a solute in a pure liquid of the solute (i.e., equivalent to a vapor pressure).

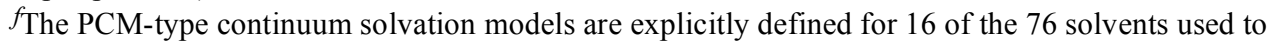
compute the self-solvation energies and the MUEs for these 16 solvents are given in parentheses.

SM6 employs an ionic training set that is more than double the size of that used for prior models. In addition, ions clustered with one water molecule are included in the training set. Because ionic solvation free energies are so sensitive to atomic Coulomb radii, a good ionic training set is very important for setting this critical parameter. By using ionic cluster data, it proved possible to do a much more thorough job of determining physically realistic Coulomb radii. The performance of the SM6 model is marginally better than SM5.43 for neutral molecules, but significantly better for ions, which makes the model more accurate for properties such as those described in more detail in Section 3 of this 
article, e.g., $\mathrm{p} K_{a}$ values and oxidation and reduction potentials. Development of organic solvation models within the SM6 framework is presently ongoing.

Another new frontier that is the subject of exploration is extension of the SM6 model to temperatures other than $298 \mathrm{~K}$. A preliminary temperature-dependent water model that is applicable to compounds containing $\mathrm{C}, \mathrm{H}$, and $\mathrm{O}$ has been described [158]; the results are very encouraging.

2.3. Vapor pressures and solubilities. Another partitioning that is of special interest is that of a solute between a solvent and a pure phase of the solute itself, i.e., pure liquid or solid solute. This is of particular interest because one may relate this free energy of partitioning of a liquid or solid solute to that solute's solubility in a given phase. If we first consider a liquid species $A_{(1)}$ in equilibrium with its own vapor $\mathrm{A}_{(\mathrm{g})}$

$$
\mathrm{A}_{(\mathrm{g})} \Leftrightarrow \mathrm{A}_{(\mathrm{l})}
$$

With a 1 molar standard-state and ideal behavior in both phases, the standard-state free energy of this process is

$$
\Delta G_{1}^{\mathrm{o}}=R T \ln \frac{P_{\mathrm{A}}^{\cdot}}{P^{\mathrm{o}} M_{\mathrm{A}}^{1}}
$$

where $R$ is the universal gas constant, $T$ is the temperature, $P_{\mathrm{A}}^{\bullet}$ is the equilibrium vapor pressure of A over pure A, $P^{\mathrm{o}}$ is the pressure $(24.45 \mathrm{~atm})$ of an ideal gas at 1 molar concentration and $298 \mathrm{~K}$, and $M_{\mathrm{A}}^{1}$ is the equilibrium molarity of the pure liquid solution of $\mathrm{A}$, which is obtained from the liquid density of A. By rearrangement of eq. (18) one may compute the vapor pressure using models that predict $\Delta G_{1}^{\mathrm{o}}$. The universal SM5-type models have been used for this purpose for a large number of cases where the necessary solvent descriptors are already available $[137,159,160]$; accuracies within a $\log$ unit are routine (cf. last line of Table 2).

Let us now consider the equilibrium between pure liquid A and an aqueous solution of A (we specify aqueous because of the importance of water, but the below analysis may be generalized to any solvent)

$$
\mathrm{A}_{(\mathrm{l})} \Leftrightarrow \mathrm{A}_{(\mathrm{aq})}
$$

assuming that all activity coefficients are unity, the standard-state free energy for the phase transfer is

$$
\Delta G_{2}^{\mathrm{o}}=-R T \ln \frac{M_{\mathrm{A}}^{\mathrm{aq}}}{M_{\mathrm{A}}^{\mathrm{l}}}
$$

where $M_{\mathrm{A}}^{\mathrm{aq}}$ is the equilibrium aqueous molarity of solute A, i.e., its solubility in molarity units, also denoted as $S$. Combining eqs. (17) and (19) gives

$$
\mathrm{A}_{(\mathrm{g})} \Leftrightarrow \mathrm{A}_{(\mathrm{aq})}
$$

The standard-state free energy change in this process is the standard-state aqueous free energy of solvation of solute $\mathrm{A}, \Delta G_{\mathrm{S}(\mathrm{aq})}^{\mathrm{o}}$; and we can calculate it by adding eqs. (18) and (20), which yields

$$
\Delta G_{\mathrm{S}(\mathrm{aq})}^{\mathrm{o}}=R T \ln \frac{P_{\mathrm{A}}^{\cdot}}{P^{\mathrm{o}}}-R T \ln M_{\mathrm{A}}^{\mathrm{aq}}
$$

Equation (22) is based on the assumption that the aqueous solution of A obeys Henry's law, i.e., that the saturated solution behaves as though infinitely dilute. In general, then, we may predict solubility from 


$$
S \equiv M_{\mathrm{A}}^{\mathrm{aq}}=\left(P_{\mathrm{A}}^{\cdot} / P^{\mathrm{o}}\right) \exp \left[-\Delta G_{\mathrm{S}(\mathrm{aq})}^{\mathrm{o}} / R T\right]
$$

where the SMx models are used to compute $P_{\mathrm{A}}^{\cdot}$ and $\Delta G_{\mathrm{S}(\mathrm{aq})}^{\mathrm{o}}$. In the case of 70 organic liquids and 13 organic solids, we obtained MUEs (log units) of less than 0.4 when using the SM5.42 model for all calculations with either AM1, HF/MIDI!, or B3LYP/MIDI! as the underlying Hamiltonian [160]. The successful prediction of the solid solubilities is particularly noteworthy as we treated these compounds essentially as supercooled liquids. That is, we estimated the necessary "solvent" descriptors for use with the universal SM5.42 model and then computed vapor pressures for the solutes in equilibrium with their solid form. The solids were for the most part aromatic hydrocarbons, and this approximation is likely to be less useful for solutes that have more specific intermolecular interactions in their crystalline solid form. Nevertheless, it suggests that the computation of solid/liquid partitioning and solubility may be reasonably started from an SMx foundation.

\section{SMx Models for Other Condensed Phases}

Homogeneous liquid solutions are arguably the simplest condensed phases to represent with a dielectric continuum model. We may, however, expect that other condensed phases having liquid-like properties, e.g., a fluid-phase lipid membrane, might be addressable with the SMx modeling approach. Inasmuch as the SM5 organic parameterizations are designed to be universal, the data required to model any phase are simply the most appropriate values of $\varepsilon, n, \gamma, \alpha$, and $\beta$ (and $\phi$ and $\psi$ if an SM5.42 or SM5.43 model is employed). Typically, of course, values for these quantities are not available. However, they may be estimated by any number of approaches (e.g., on the basis of molecular functionality also found in other molecules for which solvent parameters are available). A still more pragmatic approach for dealing with such poorly characterized systems is to treat the unknown "solvent" descriptors as being fitting parameters themselves. For all but the dielectric constant, this is particularly simple insofar as the solvation free energy depends upon them linearly. Thus, a typical approach is to make an educated guess at the dielectric constant, compute $\Delta G_{\mathrm{ENP}}^{\mathrm{o}}$ for solutes for which solvation free energies or partition coefficients (which are differences of solvation free energies between two phases, as described further below) are known, and determine the optimal values of the remaining descriptors that minimize errors in the remaining term $G_{\mathrm{CDS}}^{\mathrm{o}}$ through multilinear regression. A final model may be determined by trial-and-error optimization of $\varepsilon$.

The first example of this approach was described for a phosphatidyl choline bilayer [17]. Available experimental data were partition coefficients $P$ between the bilayer and surrounding aqueous solvent
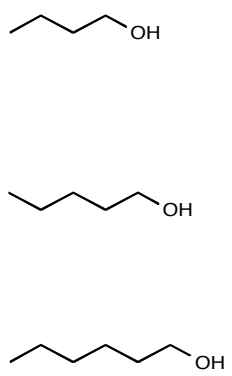<smiles>CCCCOCCO</smiles><smiles>Clc1ccccc1</smiles><smiles>Cc1cc(O)ccc1Cl</smiles><smiles>Nc1ccccc1</smiles><smiles>C=CCc1ccc(O)cc1</smiles><smiles>Nc1ccc([N+](=O)[O-])cc1</smiles><smiles>Clc1cc(Cl)cc(Cl)c1</smiles><smiles>Cc1c(Cl)cc(Cl)cc1Cl</smiles><smiles>Cc1ccc([N+](=O)[O-])cc1</smiles><smiles>Cc1ccc(O)cc1</smiles><smiles>Cc1ccccc1</smiles><smiles>Cc1ccc(C)cc1</smiles><smiles>Oc1ccc(-c2ccccc2)cc1</smiles><smiles>Nc1c(Cl)cc(Cl)cc1Cl</smiles><smiles>c1ccc2ncccc2c1</smiles>

Figure 1: Solutes used in the parameterization of an SM5.4 model for phosphatidyl choline. 
for the molecules shown in Figure 1. A partition coefficient depends on free energies of solvation according to

$$
\log P_{\mathrm{A} / \mathrm{B}}=-\frac{\Delta G_{\mathrm{S}, \mathrm{A}}^{\mathrm{o}}-\Delta G_{\mathrm{S}, \mathrm{B}}^{\mathrm{o}}}{2.303 R T}
$$

where $\mathrm{A}$ and $\mathrm{B}$ are the two phases between which a solute is partitioning, $\Delta G_{\mathrm{S}, \mathrm{X}}^{\mathrm{o}}$ is the free energy of solvation from the gas phase into phase $\mathrm{X}$ (the quantity directly computable from an SMx model), $R$ is the universal gas constant, and $T$ is temperature. Using either experimental or computed aqueous solvation free energies provides target values for the solvation free energy into the phosphatidyl choline bilayer by rearrangement of eq. (17). Assuming the dielectric constant of the bilayer to be 5.0 (an estimate based on the dielectric constant of octanol), and assuming the $\alpha$ value to be 0.0 (as there is no significant hydrogen bond donating functionality in phosphatidyl choline), an SM5.4 model was developed by regressing the target free energies of solvation on the $n, \gamma$, and $\beta$ solvent parameters. The regression provided values of $1.40,27$, and 1.15 for these parameters, which quantities are very much in the range of physically realistic values that one might have expected based on analogous molecules as solvents. In this case, the regression was also allowed to have a constant term, which was calculated to be $0.59 \log$ units. The quality of the regression, with an $R^{2}$ value of 0.80 (Figure 2), is in the range of those typically employed for predictions of bioavailability of druglike molecules, and the specificrange-parameter (SRP) SM5.4 model may be expected to be useful when used to make predictions about molecules having functionalities not too different from those included in the training set. Of course, the introduction of significantly different functionality would likely require retraining to ensure maximum predictive accuracy.

A similar phase parameterization has been accomplished for soil (i.e., dirt) [161], using a training set much larger than the phosphatidyl choline case. From an environmental perspective, an important physical parameter affecting the fate of ecosystem contaminants is the soil-water partition coefficient. Because this often depends primarily on the soil's organic carbon content, measured values are usually normalized for the organic carbon (OC) content of soil, in which case the soil sorption equilibrium constant is expressed as [162]

$$
K_{\mathrm{OC}}=\frac{C_{\mathrm{soil}} / C_{\mathrm{soil}}^{\mathrm{o}}}{C_{\mathrm{w}} / C_{\mathrm{w}}^{\mathrm{o}}}
$$

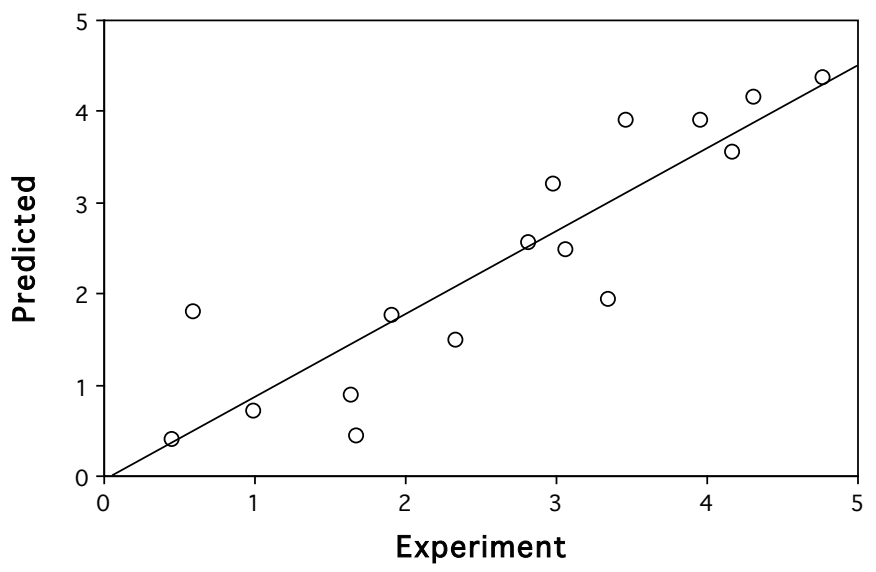

Figure 2: Predicted vs. experimental water/phosphatidyl choline partition coefficients ( $\log _{10}$ units). 
where $C_{\text {soil }}$ is the concentration of solute per gram of carbon in a standard soil, and $C_{\mathrm{w}}$ is the concentration of solute per volume of aqueous solution. The standard state concentrations $C_{\text {soil }}^{\mathrm{o}}$ and $C_{\mathrm{w}}^{\mathrm{o}}$ are typically chosen as $1 \mu \mathrm{g}$ solute per g organic carbon for soil and $1 \mu \mathrm{g}$ solute per $\mathrm{mL}$ for aqueous solution. Although this quantity is often called the soil/water equilibrium constant for simplicity, one should note that it is actually a measure of partitioning between soil organic matter and water, normalized to organic carbon content.

In this instance, a database of 387 molecules containing a wide variety of organic functional groups was used for a training set. The dielectric constant for the soil phase was optimized by trial and error to a value of $\varepsilon=15$. While so high a dielectric constant may seem unusual for a solid organic phase, it should be kept in mind that the soil used in the experiment is "wet", i.e., it is in contact with the aqueous phase and swelled by it. The optimized dielectric constant was used for the computation of electrostatics at the SM5.42/HF/MIDI!/HF/MIDI! and SM5.42/AM1//AM1 levels.

Errors in residual $G_{\mathrm{CDS}}^{\mathrm{o}}$ values were minimized by fitting the $n, \gamma, \alpha$, and $\beta$ solvent parameters as described above. For the SM5.42/HF/MIDI! level, these parameters took on values of $1.311,45.3,0.56$, and 0.60 ; such values are consistent with the functionalities expected for the organic component of soil (which include aromatics, phenols, quinones, and carboxylic acids). The resulting soil partitioning model had a MUE over the training set of $0.98 \mathrm{kcal} / \mathrm{mol}$.

A point that merits emphasis is that application of the SMx models to predict solvation or partitioning behavior can certainly be useful as a predictive exercise, but a potentially still more important feature of the models is that the free energy associated with the particular phase transfer can be decomposed into atomic or group contributions, and thus interpreted in a fragment basis [163,164]. The general form of the GB and surface tension equations should make this point clear: $\Delta G_{\mathrm{ENP}}^{\mathrm{o}}$ is computed as a sum of atomic-charge dependent terms, and $G_{\mathrm{CDS}}^{\mathrm{o}}$ is computed as a sum over atomic solvent-exposed surface areas.

For instance, Figure 3 illustrates the correlation between experimental and predicted $\log K_{\mathrm{OC}}$ values for polychlorinated biphenyls (PCBs). There is a systematic error of about 1 log unit in the absolute accuracy of the predictions, but otherwise a fairly good correlation $\left(R^{2}=0.86\right)$. Analysis of atomic contributions indicates that chlorine atoms near the biphenyl ipso carbons are less hydrophilic (owing to decreased exposure to the solvent) than chlorine atoms substituted at other positions. Such fragment analysis can be useful in drug design, where particular ranges of partitioning constants may enhance drug bioavailability.

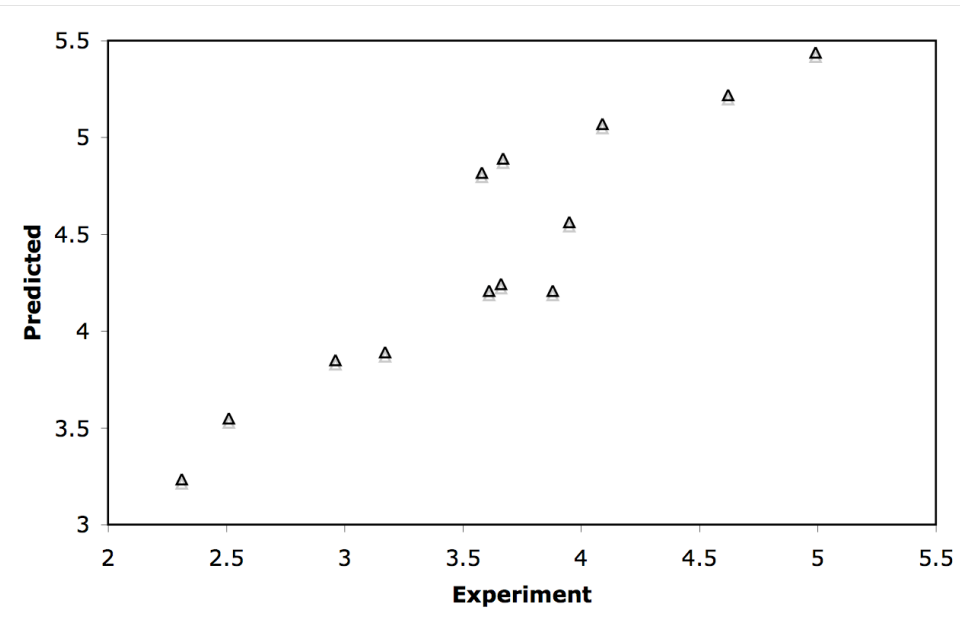

Figure 3: Predicted vs. experimental soil/water partition coefficients for PCBs ( $\log _{10}$ units). 
A final example of a novel phase to which the SMx models have been extended is the surface layer of an aqueous solution that is in contact with air, i.e., the air-water interface. This is a phase that is formally two-dimensional, although at the molecular level it has some non-zero thickness. In this case the measured equilibrium constant of interest is

$$
K_{\mathrm{i} / \mathrm{a}}=\frac{\Gamma_{i}}{C_{\mathrm{a}}}
$$

where $\Gamma_{\mathrm{i}}$ is the concentration of the adsorbed solute at the air-water interface in $\mathrm{mol} / \mathrm{m}^{2}$, and $C_{\mathrm{a}}$ is the equilibrium vapor concentration in $\mathrm{mol} / \mathrm{m}^{3}$ of the solute. The adsorption coefficient for a molecule transferring from the gas phase to a air-water interface is related to the standard-state free energy of transfer by:

$$
\ln K_{\mathrm{i} / \mathrm{a}}=-\frac{\Delta G_{\mathrm{i} / \mathrm{a}}^{\circ}}{R T}
$$

However, the free energy in eq. (27) differs from all of the other free energies that have thus far been described in the sense that it contains two distinct components. The first component is the coupling free energy associated with the new interactions that a molecule in a condensed phase has in comparison to the gas phase - this is the component that the SMx models are designed to predict. In the case of $\Delta G_{\mathrm{i} / \mathrm{a}}^{\circ}$, however, there is also a term associated with the loss of entropy associated with moving from a phase of 3 dimensions to one of 2 dimensions. That is

$$
\Delta G_{\mathrm{i} / \mathrm{a}}^{\circ}=\Delta G_{\text {coup }(\mathrm{a} \rightarrow \mathrm{i})}^{\mathrm{o}}+\Delta G_{3 \mathrm{D} \rightarrow 2 \mathrm{D}}^{\circ}
$$

The coupling term may be computed directly, however, and depends only on the molecular weight. Thus, a particle of mass $m$ at temperature $T$ has a de Broglie wavelength equal to [165]

$$
\Lambda=\sqrt{\frac{h^{2}}{2 \pi m k T}}
$$

where $k$ is Boltzmann's constant, and $h$ is Planck's constant. For an ideal gas molecule in $M$ dimensions, the molecular translational partition function can be written as [165]

$$
q=\left(\frac{L^{\circ}}{\Lambda}\right)^{M}
$$

where $L^{\circ}$ is the standard-state unit of length. The molar translational partition function is then

$$
Z=\frac{1}{N_{A} !}\left(\frac{L^{\circ}}{\Lambda}\right)^{M N_{A}}
$$

where $N_{A}$ is Avagadro's number. With this partition function and taking standard thermodynamic relationships for internal energy $U$, enthalpy $H$, and entropy $S$ as a function of $Z$ [165], we may compute

$$
U^{\circ}=\frac{M}{2} R T \quad H^{\circ}=U^{\circ}+P V \quad S^{\circ}=R \ln \left[\frac{e^{(M+2) / 2}}{N}\left(\frac{L^{\circ}}{\Lambda}\right)^{M}\right]
$$

and thus, from

$$
\Delta G_{3 \mathrm{D} \rightarrow 2 \mathrm{D}}^{\circ}=\Delta H_{3 \mathrm{D} \rightarrow 2 \mathrm{D}}^{\circ}-T \Delta S_{3 \mathrm{D} \rightarrow 2 \mathrm{D}}^{\circ}
$$


we derive

$$
\Delta G_{3 D \rightarrow 2 D}^{\circ}=\Delta(P V)_{3 \mathrm{D} \rightarrow 2 \mathrm{D}}-R T \ln \left(\frac{\Lambda}{L^{\circ}}\right)
$$

The $P V$ terms may be calculated from the microcanonical relationship [165]

$$
\frac{P}{T}=\left\{\frac{\partial S}{\partial\left[\left(L^{\circ}\right)^{M}\right]}\right\}_{N, U}
$$

Using $S^{\mathrm{o}}$ as defined in eq. (32) gives

$$
\frac{P}{T}=\frac{R}{\left(L^{\circ}\right)^{M}}
$$

which is the ideal gas law $P V=R T$ but expressed for volume in an arbitrary number of dimensions. Thus, $\Delta P V$ is zero for the dimensionality change and we have

$$
\Delta G_{3 D \rightarrow 2 D}^{\circ}=-R T \ln \left(\frac{\Lambda}{L^{\circ}}\right)
$$

When this component is removed from measured free energies of adsorption, the remaining free energies of coupling can be used as a training set for the determination of an air-water interface SM $x$ model in the usual way.

In this case, however, there is some ambiguity associated with computing the electrostatic component of solvation. One approximation might be to assume that a molecule at the interface has half the value of $\Delta G_{\mathrm{ENP}}^{\mathrm{o}}$ that would be computed in the bulk. A more accurate model would account for preferential orientation of surface molecules. In previous work, however, we instead investigated whether an SM5.0 model (i.e., one that ignores electrostatics) would be successful in making predictions. For a training set of 85 solutes, we found that a best fit of the "solvent" descriptors for the air-water interface involved taking $\alpha, \beta$, and $\gamma$ to be $1.11,0.59$, and -144.6 , respectively [166].

These values appear to be quite physical. Various prior simulations and experimental studies [167-170] have suggested that dangling hydrogen bond sites at the air-water interface make surface water more active as a hydrogen bond donor and acceptor than bulk water (which has $\alpha$ and $\beta$ values of 0.82 and 0.35 , respectively [171]). The negative value for the surface tension is also reasonable, since for the adsorption process no free energy is being expended in order to cavitate, but instead new dispersion interactions are being added by the solute "sticking" to the surface that were not present previously. The SM5.0surf model has a MUE (log units) of 0.47 for the 85 molecules in the training set [166], many of which are functionally rich pesticides and other environmental contaminants. 


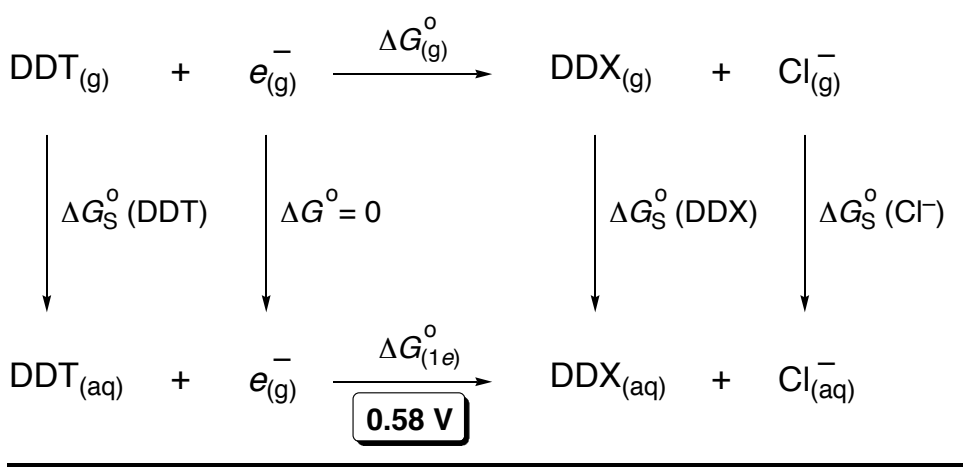

$\mathrm{DDT}_{(\mathrm{g})}+\mathrm{H}_{(\mathrm{g})}^{+}+2 e_{(\mathrm{g})}^{-} \stackrel{\Delta G_{(\mathrm{g})}^{\circ}}{\longrightarrow} \mathrm{DDD}_{(\mathrm{g})}+\mathrm{Cl}_{(\mathrm{g})}^{-}$
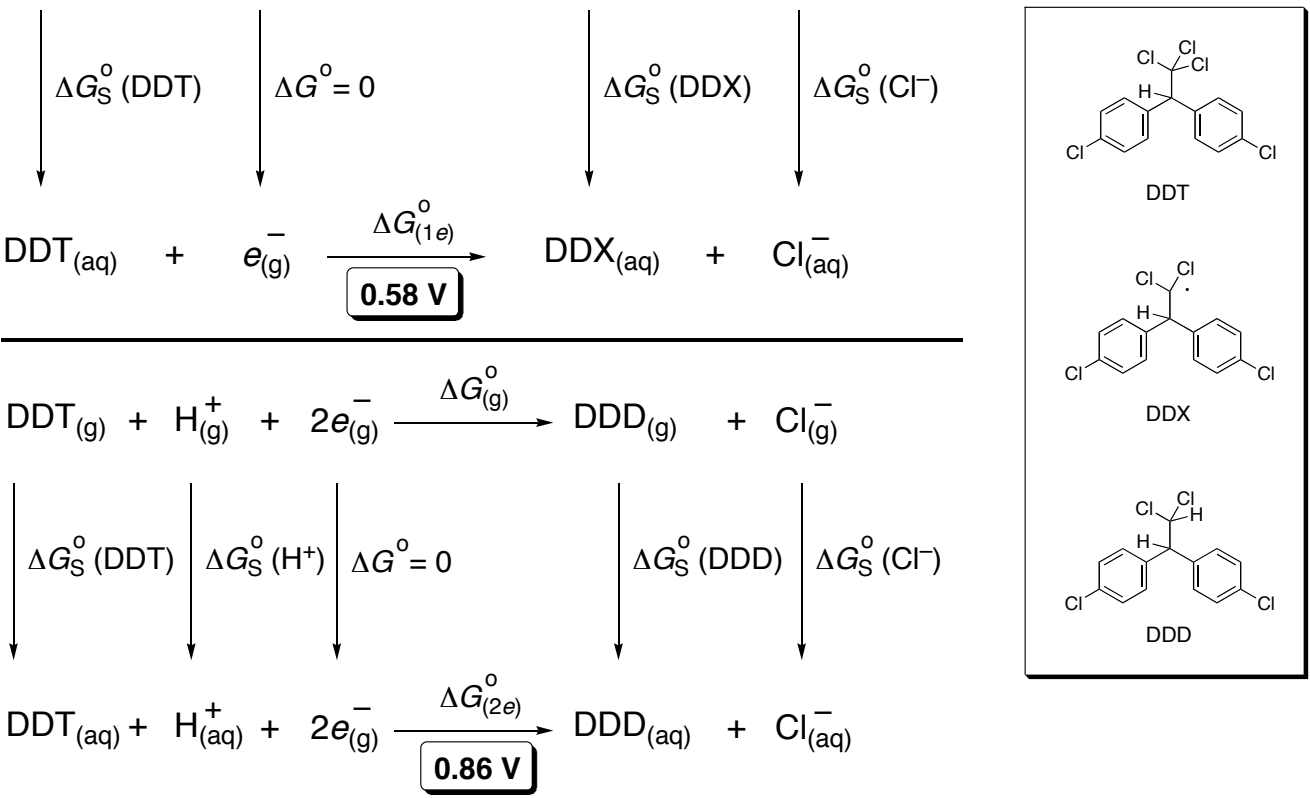

Figure 4: One- and two-electron reduction half reactions for DDT.

\section{Selected Applications in Environmental Chemistry}

Electron-transfer reactions play important roles in many pathways that degrade contaminants in the environment. Such electron transfers are often described with electrochemical half reactions, like those in Figure 4 for the one- and two-electron reductions of the pesticide DDT.

In order to compute the lower leg of each thermodynamic cycle, it is sufficient to compute the upper leg, which is a gas phase process, at some suitable level of electronic structure theory, and then to add the vertical legs, which quantify the differences between the solvation free energies of the various reagents. In the case of the reactions in Figure 4, calculations were carried out at the B3LYP/6$311+\mathrm{G}(\mathrm{d}) / / \mathrm{B} 3 \mathrm{LYP} / 6-31 \mathrm{G}(\mathrm{d})$ level for gas-phase energetics, the solvation free energies of the proton and chloride anion were taken from experiment [172], and the remaining solvation free energies were computed at the SM5.42/BPW91/6-31G(d)//B3LYP/6-31G(d) level [173].

The resulting free energy of reaction for a solution process $\Delta G_{\mathrm{rxn}}^{\mathrm{o}}$ may be converted to a potential relative to the normal hydrogen electrode (NHE) by

$$
E^{\mathrm{o}}=\frac{\Delta G_{\mathrm{rxn}}^{\mathrm{o}}-\Delta G_{\mathrm{NHE}}^{\mathrm{o}}}{n F}
$$

where $n$ is the number of electrons transferred, $F$ is the Faraday constant in appropriate units, and $\Delta G_{\mathrm{NHE}}^{\mathrm{o}}$ is the free energy of reaction for the reference half reaction

$$
\frac{1}{2} \mathrm{H}_{2(\mathrm{~g})} \rightarrow \mathrm{H}_{(\mathrm{aq})}^{+}+e_{(\mathrm{g})}^{-}
$$

which is $4.28 \mathrm{eV}[173,174]$. Note that this value, and the values listed in Figure 4, differ from those reported in the original reference [173] because of (i) a standard-state error made in the original reference for the solvation free energy of the proton, as explained in subsequent work [174], an 


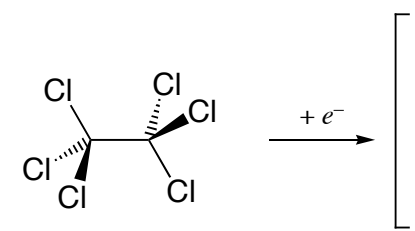

0.0<smiles>ClC(Cl)(Cl)C(Cl)(Cl)Cl</smiles>

no barrier<smiles>ClC(Cl)C(Cl)(Cl)Cl</smiles><smiles>ClC(Cl)C(Cl)(Cl)Cl</smiles>

or<smiles>ClC(Cl)C(Cl)(Cl)Cl</smiles>

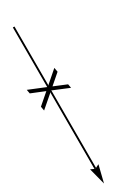

no barrier
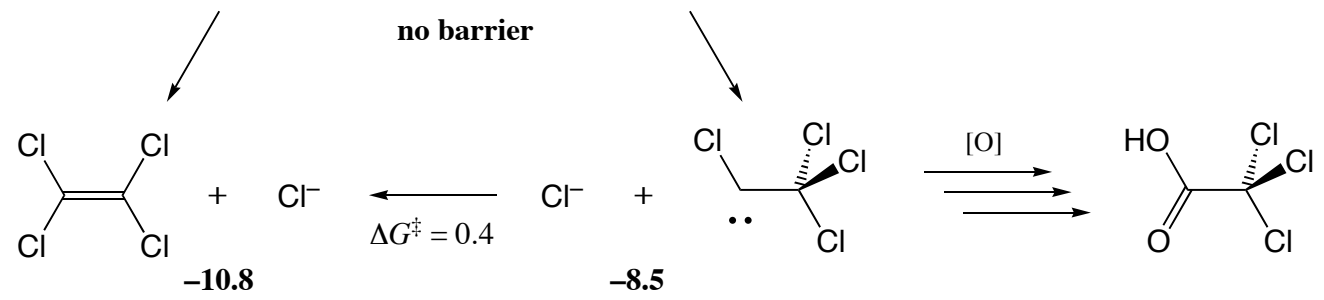

Figure 5: Steps in the reductive dechlorination of hexachloroethane in aqueous solution. Relative energies of stoichiometrically equivalent species are given in $\mathrm{eV}$.

error in the $298 \mathrm{~K}$ free energy of the chloride anion (which should have a tabulated value of -460.31875 $E_{\mathrm{h}}$ in the original reference [173]), and (iii) the use here of the experimental solvation free energy for the chloride anion instead of a computed solvation free energy (a difference of $0.1 \mathrm{eV}$ ).

Reduction (and oxidation) potentials are useful quantities for analyzing environmental persistence in phases having either oxidizing or reducing character. Indeed, taken together with soil/water partition coefficients, which may also be computed using SM $x$ methods, they are key components in the prediction of environmental fate constants for organic contaminants.

Solvation free energies can also be added to reagents in reactions that do not involve electron transfer. Accounting for and predicting changes in reaction pathways induced by solvation is a key application of solvation models. One interesting mechanism that we have studied [175], which involves both electron transfer steps and steps in which electrons do not appear as individual species, is the reductive dechlorination of hexachloroethane (Figure 5).

In the initial reductive dechlorination, loss of the first chloride anion is predicted to proceed without barrier subsequent to electron transfer in aqueous solution. One proposal that had been made in the literature was that a heterolytic ionization of the resulting radical might precede a second electron transfer, however this step is predicted at the SM5.42/BPW91/aug-cc-pVDZ//BPW91/aug-cc-pVDZ level of theory to be too endergonic to be important. Interestingly, chloride elimination is again predicted to proceed without barrier following the second electron transfer. However, that prediction holds true for both sets of stereochemically distinct chlorine atoms, those leading to the much more stable tetrachloroethene and those leading to the less stable chlorotrichloromethylcarbene.

Rearrangement of the carbene is predicted to have a sufficiently high barrier that diffusion-controlled bimolecular processes might compete with rearrangement of the singlet carbene to the alkene. One such process involves attack of water on the carbene to generate an oxonium ylide that, upon 
rearrangement would generate a chlorohydrin that could subsequently be easily oxidized to trichloromethylacetic acid. The latter species is detected in small amounts in the reductive

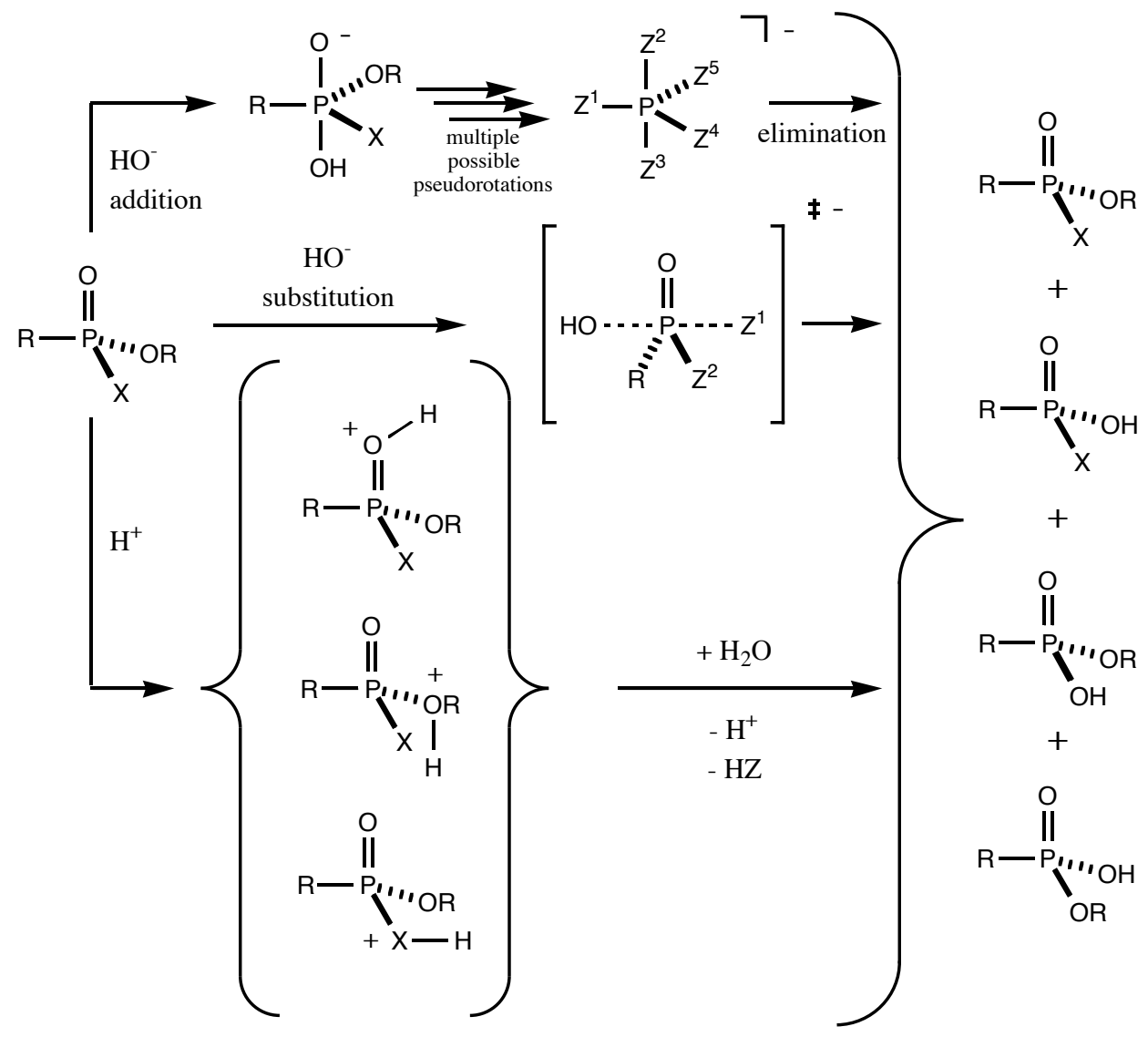

Figure 6: Possible pathways for aqueous hydrolysis of a generic phosphonate derivative.

dechlorination process, and the mechanism of its formation had not been well understood prior to our modeling efforts [175].

The prediction of $\mathrm{p} K_{\mathrm{a}}$ values for environmental contaminants can also be particularly important because of the potentially enhanced (or retarded) activity of molecules in their conjugate acid or base forms. In the case of phosphorus-based chemical weapons agents, for example, aqueous decontamination can follow different reaction coordinates depending on aqueous $\mathrm{pH}$ (Figure 6).

One mechanistic pathways is the reaction of the weapons agent with hydroxide acting as a nucleophile. In the upper pathway, the addition of hydroxide to phosphorus leads to a phosphorane anion intermediate. In general, the elimination of ligands from phosphoranes is more facile from apical positions than from equatorial. Stereochemistry in the phosphorane, i.e., the energetic preference for location of individual groups in apical or equatorial positions, is controlled by a number of factors, including apicophilicity and hyperconjugative interactions between the ligands [176] and solvation effects. Interconversion between different trigonal bipyramids requires a pseudorotation, and the barriers to pseudorotation in such complex phosphoranes are not well understood and may also be subject to solvation effects.

An alternative pathway (the central one in Figure 6) is a one-step bimolecular substitution. Depending on the substituents of the original phosphonate, either or both of these two pathways may be operating. 
In general, the direct substitution pathway becomes more favorable when the leaving group is the conjugate base of a strong acid.

Finally, under neutral or acidic conditions, the nucleophile may not be hydroxide but instead a water molecule (the lower path in the figure). Under these conditions, there are again two paths, addition/elimination and direct substitution, either or both of which are potentially operative.

The SM6 aqueous solvation model has recently been demonstrated to be particularly effective for the computation of $\mathrm{p} K_{\mathrm{a}}$ values [177] (although they are still subject to errors of 1 to $2 \mathrm{p} K$ units in unfavorable cases). Other SM $x$ models have also been decisive in explaining key features in the hydrolytic detoxification of chemical weapons agents. For example, Seckute et al. [178] were able to explain the anomalous reactivity of VX-like compounds (thiophosphonates having potential neurotoxic properties) with aqueous hydroperoxide vs hydroxide, a case where solvation effects played a key role in favoring a beneficial reaction path (formation of an innocuous product) over a less useful one (formation of a long-lived toxic product).

\section{Conclusions}

Continuum solvation models are a critical element in the computational chemist's toolbox because of their utility for describing (i) condensed-phase effects on molecular structure and properties and (ii) the connection between gas-phase and condensed-phase potential energy surfaces. The parameterization of the SM $x$ models against a large and diverse set of data, the broad variety of electronic structure levels with which they are compatible, e.g., semiempirical theory, ab initio Hartree-Fock theory, and density functional theory, and their incorporation into several general-purpose electronic structure packages makes them particularly attractive for modeling. Future developments designed to include temperature dependence, improve accuracy, and extend the range of condensed phases to which they may be applied, may be expected to further increase their general utility.

\section{Acknowledgments}

The authors are grateful to Daniel Liotard, David Giesen, Gregory Hawkins, Joey Storer, Candee Chambers, Jiabo Li, Tony Zhu, Paul Winget, Jason Thompson, James Xidos, Casey Kelly, and Adam Chamberlin for extensive contributions to the development of the SMx models and to Eric Weber, Eric Patterson, and Bill Arnold for collaboration on their application to environmental problems. We are also grateful to our many co-workers on the SMx models over the years, whose names grace the publications resulting from their efforts. This work was supported by the NIH training grant for Neurophysical-computational Sciences, by the U.S. Army Research Office under Multidisciplinary Research Program of the University Research Initiative (MURI) through grant number DAAD19-02-1-0176, by the Minnesota Partnership for Biotechnology and Medical Genomics, by the National Science Foundation (CHE02-03446 and CHE03-49122), and by the Office of Naval Research under grant no. N 00014-05-01-0538.

\section{References}

[1] J. Tomasi and M. Persico, Molecular interactions in solution: An overview of methods based on continuous distributions of the solvent, Chemical Reviews 94 2027-2094(1994).

[2] C.J. Cramer and D.G. Truhlar, Development and biological applications of quantum mechanical continuum solvation models, Quantitative Treatments of Solute/Solvent Interactions, P. Politzer and J.S. Murray (Eds.), Elsevier, Amsterdam, 1994, pp. 9-54.

[3] C.J. Cramer and D.G. Truhlar, Continuum solvation models: Classical and quantum mechanical implementations, Reviews in Computational Chemistry, K.B. Lipkowitz and D.B. Boyd (Eds.), VCH, New York, 1995, pp. 1-72.

[4] J.-L. Rivail and D. Rinaldi, Liquid state quantum chemistry: Computational applications of the polarizable continuum models, Computational Chemistry, Review of Current Trends, J. Leszczynski (Ed.), World Scientific, New York, 1996, pp. 139-174. 
[5] C.J. Cramer and D.G. Truhlar, Continuum solvation models, Solvent Effects and Chemical Reactivity, O. Tapia and J. Bertrán (Eds.), Kluwer, Dordrecht, 1996, pp. 1-81.

[6] C.J. Cramer and D.G. Truhlar, Implicit solvation models: Equilibria, structure, spectra, and dynamics, Chemical Reviews 99 2161-2200(1999).

[7] M. Orozco and F.J. Luque, Theoretical methods for the description of the solvent effect on biomolecular systems, Chemical Reviews 100 4187-4225(2000).

[8] D. Bashford and D.A. Case, Generalized Born models of macromolecular solvation effects, Annual Review of Physical Chemistry 51 129-152(2000).

[9] C.J. Cramer and D.G. Truhlar, Thermodynamics of solvation and the treatment of equilibrium and nonequilibrium solvation effects by models based on collective solvent coordinates, Free Energy Calculations in Rational Drug Design, M.R. Reddy and M.D. Erion (Eds.), Kluwer Academic/Plenum, New York, 2001, pp. 63-95.

[10] C.J. Cramer, Essentials of Computational Chemistry: Theories and Models, John Wiley \& Sons, Chichester, 2004.

[11] J. Tomasi, Thirty years of continuum solvation chemistry: A review, and prospects for the near future, Theoretical Chemistry Accounts 112 184-203(2004).

[12] M. Feig and C.L. Brooks, Recent advances in the development and application of implicit solvent models in biomolecule simulations, Current Opinion in Structural Biology 14217 224(2004).

[13] J. Tomasi, B. Mennucci, and R. Cammi, Quantum mechanical continuum solvation models, Chemical Reviews 105 2999-3093(2005).

[14] J.W. Storer, D.J. Giesen, G.D. Hawkins, G.C. Lynch, C.J. Cramer, D.G. Truhlar, and D.A. Liotard, Solvation modeling in aqueous and nonaqueous solvents: New techniques and a reexamination of the claisen rearrangement, Structure and Reactivity in Aqueous Solution, C.J. Cramer and D.G. Truhlar (Eds.), American Chemical Society, Washington, DC, 1994, pp. $24-$ 49.

[15] D.J. Giesen, C.C. Chambers, G.D. Hawkins, C.J. Cramer, and D.G. Truhlar, Modeling free energies of solvation and transfer, Computational Thermochemistry: Prediction and Estimation of Molecular Thermodynamics, K. Irikura and D.J. Frurip (Eds.), American Chemical Society, Washington, DC, 1998, pp. 285-300.

[16] G.D. Hawkins, J. Li, T. Zhu, C.C. Chambers, D.J. Giesen, D.A. Liotard, C.J. Cramer, and D.G. Truhlar, Universal solvation models, Combined Quantum Mechanical and Molecular Mechanical Methods, J. Gao and M.A. Thompson (Eds.), American Chemical Society, Washington, DC, 1998, pp. 201-219.

[17] C.C. Chambers, D.J. Giesen, G.D. Hawkins, W.H.J. Vaes, C.J. Cramer, and D.G. Truhlar, Modeling the effect of solvation on structure, reactivity, and partitioning of organic solutes: Utility in drug design, Rational Drug Design, D.G. Truhlar, W.J. Howe, A.J. Hopfinger, J.M. Blaney, and R.A. Dammkoehler (Eds.), Springer, New York, 1999, pp. 51-72.

[18] G.D. Hawkins, J. Li, T. Zhu, C.C. Chambers, D.J. Giesen, D.A. Liotard, C.J. Cramer, and D.G. Truhlar, New tools for rational drug design, Rational Drug Design, A.L. Parrill and M.R. Reddy (Eds.), American Chemical Society, Washington, DC, 1999, pp. 120-140.

[19] C.J. Cramer and D.G. Truhlar, General parameterized scf model for free energies of solvation in aqueous solution, Journal of the American Chemical Society 113 8305-8311(1991).

[20] G.J. Hoijtink, E. de Boer, P.H. Van der Meij, and W.P. Weijland, Potentials of various aromatic hydrocarbons, Recueil de Travail Chimique des Pays-Bas 75 487-503(1956).

[21] F. Peradejordi, On the Pariser and Parr semiempirical method for computing molecular wave functions. The basic strength of N-heteroatomic compounds and their monoamines, Cahiers Physique 17 393-447(1963).

[22] I. Jano, Sur l'énergie de solvatation, Comptes Rendu Academie de Sciences, Paris 261 103105(1965).

[23] O. Tapia, Local field representation of surrounding medium effects. From liquid solvent to protein core effects, Quantum Theory of Chemical Reactions, R. Daudel, A. Pullman, L. Salem, and A. Viellard (Eds.), Reidel, Dordrecht, 1980, pp. 25-72.

[24] S.C. Tucker and D.G. Truhlar, Generalized Born fragment charge model for solvation effects as a function of reaction coordinate, Chemical Physics Letters 157 164-170(1989).

[25] W.C. Still, A. Tempczyk, R.C. Hawley, and T. Hendrickson, Semianalytical treatment of solvation for molecular mechanics and dynamics, Journal of the American Chemical Society 112 6127-6129(1990). 
[26] C.J. Cramer and D.G. Truhlar, AM1-SM2 and PM3-SM3 parameterized scf solvation models for free energies in aqueous solution, Journal of Computer-Aided Molecular Design 6 629666(1992).

[27] O. Kikuchi, T. Matsuoka, H. Sawahata, and O. Takahashi, Ab initio molecular orbital calculations including solvent effects by generalized Born formula. Conformation of zwitterionic forms of glycine, alanine, and serine in water, Journal of Molecular Structure (Theochem) 305 79-87(1994).

[28] D.A. Liotard, G.D. Hawkins, G.C. Lynch, C.J. Cramer, and D.G. Truhlar, Improved methods for semiempirical solvation models, Journal of Computational Chemistry 16 422-440(1995).

[29] S.L. Chan and C. Lim, Reducing the error due to the uncertainty in the Born radius in continuum dielectric calculations, Journal of Physical Chemistry 98 692-695(1994).

[30] G.D. Hawkins, C.J. Cramer, and D.G. Truhlar, Pairwise solute screening of solute charges from a dielectric medium, Chemical Physics Letters 246 122-129(1995).

[31] D. Qiu, P.S. Shenkin, F.P. Hollinger, and W.C. Still, The GB/SA continuum model for solvation. A fast analytical method for the calculation of approximate Born radii, Journal of Physical Chemistry A 101 3005-3014(1997).

[32] B. Jayaram, Y. Liu, and D.L. Beveridge, A modification of the generalized Born theory for improved estimates of solvation energies and pK shifts, Journal of Chemical Physics 109 1465-1471(1998).

[33] C.S. Babu and C. Lim, A new interpretation of the effective Born radius from simulation and experiment, Chemical Physics Letters 310 225-228(1999).

[34] C.S. Babu and C. Lim, Incorporating nonlinear solvent response in continuum dielectric models using a two-sphere description of the Born radius, Journal of Physical Chemistry 105 5030-5036(2001).

[35] A. Onufriev, D.A. Case, and D. Bashford, Effective Born radii in the generalized Born approximation: The importance of being perfect, Journal of Computational Chemistry 23 1297-1304(2002).

[36] M.S. Lee, F.R. Salsbury, and C.L. Brooks, Novel generalized Born methods, Journal of Chemical Physics 116 10606-10614(2002).

[37] B. Lee and F.M. Richards, The interpretation of protein structure: Estimation of static accessibility, Journal of Molecular Biology 55 379-400(1971).

[38] J.L. Pascual-Ahuir and E. Silla, GEPOL: An improved description of molecular surfaces. I. Building the spherical surface set, Journal of Computational Chemistry 11 1047-1059(1990).

[39] M.J.S. Dewar, E.G. Zoebisch, E.F. Healy, and J.J.P. Stewart, AM1: A new general purpose quantum mechanical molecular model, Journal of the American Chemical Society $1073902-$ 3909(1985).

[40] R.S. Mulliken, Electronic population analysis on LCAO-MO molecular wave functions. I., Journal of Chemical Physics 23 1833-1840(1955).

[41] C.J. Cramer and D.G. Truhlar, An SCF solvation model for the hydrophobic effect and absolute free energies of aqueous solvation including specific water interactions, Science (Washington DC) 256 213-217(1992).

[42] C.J. Cramer and D.G. Truhlar, PM3-SM3: A general parameterization for including aqueous solvation effects in the PM3 molecular orbital model, Journal of Computational Chemistry 13 1089-1097(1992).

[43] J.J.P. Stewart, Optimization of parameters for semiempirical methods. 1. Method, Journal of Computational Chemistry 10 209-220(1989).

[44] D.R. Armstrong, R. Fortune, P.G. Perkins, and J.J.P. Stewart, Molecular orbital theory for the excited states of transition metal complexes, Journal of the Chemical Society, Faraday Transactions 268 1839-1846(1972).

[45] D.R. Armstrong, P.G. Perkins, and J.J.P. Stewart, Bond indices and valency, Journal of the Chemical Society, Dalton Transactions 838-840(1973).

[46] G.D. Hawkins, D.J. Giesen, G.C. Lynch, C.C. Chambers, I. Rossi, J.W. Storer, J. Li, J.D. Thompson, P. Winget, B.J. Lynch, D. Rinaldi, D.A. Liotard, C.J. Cramer, and D.G. Truhlar, Amsol-version 7.1, University of Minnesota, Minneapolis, MN, 2003.

[47] Spartan version 5, Wavefunction, Inc., Irvine, CA, 1998.

[48] A. Acker, H.J. Hoffmann, and R. Cimiraglia, On the tautomerism of maleimide and phthalimide derivatives, Journal of Molecular Structure (Theochem) 121 43-51(1994). 
[49] B. Hernández, M. Orozco, and F.J. Luque, Tautomerism of xanthine and alloxanthine: A model for substrate recognition by xanthine oxidase, Journal of Computer-Aided Molecular Design 10 535-544(1996).

[50] F. Lombardo, J.F. Blake, and W.J. Curatolo, Computation of brain-blood partitioning of organic solutes via free energy calculations, Journal of Medicinal Chemistry 394750 4755(1996).

[51] J. Wang and R.J. Boyd, A theoretical study of proton transfers in aqueous para-, orthohydroxypyridine and para-, ortho-hydroxyquinoline, Chemical Physics Letters 259647 653(1996).

[52] G.D. Hawkins, C.J. Cramer, and D.G. Truhlar, New methods for potential functions for simulating biological molecules, Journal de Chimie Physique 94 1448-1481(1997).

[53] I. Lee, C.K. Kim, B.-S. Lee, C.K. Kim, H.W. Lee, and I.S. Han, Theoretical studies on the transition-state imbalance in malononitrile anion-forming reactions in the gas phase and in water, Journal of Physical Organic Chemistry 10 908-916(1997).

[54] F.C. Lightstone and T.C. Bruice, Separation of ground state and transition state effects in intramolecular and enzymatic reactions. 2. A theoretical study of the formation of transition states in cyclic anhydride formation, Journal of the American Chemical Society 119 91039113(1997).

[55] H. Adalsteinsson and T.C. Bruice, What is the mechanism of catalysis of ester aminolysis by weak amine bases? Comparison of experimental studies and theoretical investigation of the aminolysis of substituted phenyl esters of quinoline-6- and -8-carboxylic acids, Journal of the American Chemical Society 120 3440-3447(1998).

[56] M. Agback, S. Lunell, A. Hussenius, and O. Matsson, Theoretical studies of proton transfer reactions in 1-methylindene, Acta Chemica Scandanivica 52 541-548(1998).

[57] C. Aleman, H.M. Ishiki, E.A. Armelin, O. Abrahao, and S.E. Galembeck, Free energies of solvation for peptides and polypeptides using scrf methods, Chemical Physics 23385 96(1998).

[58] J.R. Alvarez-Idaboya and E.S. Kryachko, Nonrigidity of molecules in solvent and its impact on infrared spectrum: Substituted phenols and trimethylamine $N$-oxide. I., Journal of Molecular Structure (Theochem) 433 263-278(1998).

[59] A. Bagno, E. Menna, E. Mezzina, G. Scorrano, and D. Spinelli, Site of protonation of alkyland arylhydrazines probed by ${ }^{14} \mathrm{~N},{ }^{15} \mathrm{~N}$, and ${ }^{13} \mathrm{C}$ NMR relaxation and quantum chemical calculations, Journal of Physical Chemistry A 102 2888-2892(1998).

[60] M. Bräuer, M. Mosquera, J.L. Pérez-Lustres, and F. Rodríguez-Prieto, Ground-state tautomerism and excited-state proton transfer processes in 4,5-dimethyl-2-(2'hydroxyphenyl)imidazole in solution: Fluorescence spectroscopy and quantum mechanical calculations, Journal of Physical Chemistry A 102 10736-10745(1998).

[61] F. Döring, J. Will, S. Amasheh, W. Clauss, H. Ahlbrecht, and H. Daniel, Minimal molecular determinants of substrates for recognition by the intestinal peptide transporter, Journal of Biological Chemistry 273 23211-23218(1998).

[62] J. Köhler, M. Hohla, R. Sollner, and M. Amann, The difference between cholesterol- and glycyrrhizin- $\gamma$-cyclodextrin complexes - an analysis by MD simulations in vacuo and in aquo and the calculation of solvation free energies with AMSOL, Supramolecular Science 5 117137(1998).

[63] J. Köhler, M. Hohla, R. Sollner, and H.J. Eberle, Cyclohexadecanone derivative gammacyclodextrin complexes md simulations and amsol calculations in vacuo and in aquo compared with experimental findings, Supramolecular Science 5 101-116(1998).

[64] E.S. Kryachko and G. Zundel, Quantum chemical study of 1-methyladenine and its spectra in gas phase and in solvent. I., Journal of Molecular Structure (Theochem) 446 41-54(1998).

[65] S.F. Lieske, B. Yang, M.E. Eldefrawi, A.D. MacKerell, and J. Wright, (-)3 $\beta$-substituted ecgonine methyl esters as inhibitors for cocaine binding and dopamine uptake, Journal of Medicinal Chemistry 41 864-876(1998).

[66] D.J. Price, J.D. Roberts, and W.L. Jorgensen, Conformational complexity of succinic acid and its monoanion in the gas phase and in solution: Ab initio calculations and monte carlo simulations, Journal of the American Chemical Society 120 9672-9679(1998).

[67] G. Reinwald and I. Zimmermann, A combined calorimetric and semiempirical quantum chemical approach to describe the solution thermodynamics of drugs, Journal of Pharmaceutical Science 87 745-750(1998). 
[68] B. Schiøtt, Y.-J. Zheng, and T.C. Bruice, Theoretical investigation of the hydride transfer from formate to $\mathrm{NAD}^{+}$and the implications for the catalytic mechanism of formate dehydrogenase, Journal of the American Chemical Society 120 7192-7200(1998).

[69] G. Schüürmann, Quantum chemical analysis of the energy of proton transfer from phenol and chlorophenols to $\mathrm{H}_{2} \mathrm{O}$ in the gas phase and in aqueous solution, Journal of Chemical Physics 109 9523-9528(1998).

[70] M. Hoffmann, J. Rychlewski, and U. Rychlewska, Effects of substitution of OH group by F atom for conformational preferences of fluorine-substituted analogues of $(R R)$-tartaric acid, its dimethyl diester, diamide, and $N, N, N^{\prime}, N^{\prime}$-tetramethyl diamide. Ab initio conformational analysis, Journal of the American Chemical Society 121 1912-1921(1999).

[71] P.E. Just, K.I. Chane-Ching, J.C. Lacroix, and P.C. Lacaze, Anodic oxidation of dipyrrolyls linked with conjugated spacers: Study of electronic interactions between the polypyrrole chain and the spacers, Journal of Electroanalytical Chemistry 479 3-11(1999).

[72] O. Kwon, M.L. McKee, and R.M. Metzger, Theoretical calculations of methylquinolinium tricyanoquinodimethanide (CH3Q-3CNQ) using a solvation model, Chemical Physics Letters 313 321-331(1999).

[73] P.-T. Chou, G.-R. Wu, C.-Y. Wei, C.-C. Cheng, C.-P. Chang, and F.-T. Hung, Photoinduced double proton tautomerism in 4-azabenzimidazole, Journal of Physical Chemistry B $\mathbf{1 0 3}$ 10042-10052(1999).

[74] S.D. Rychnovsky, R. Vaidyanathan, T. Beauchamp, R. Lin, and P.J. Farmer, AM1-SM2 calculations model the redox potential of nitroxyl radicals such as TEMPO, Journal of Organic Chemistry 646745 -6749(1999).

[75] A.G. Turjanski, R.E. Rosenstein, and D.A. Estrin, Solvation and conformational properties of melatonin: A computational study, Journal of Molecular Modeling 5 271-280(1999).

[76] I.J. Chen and A.D. MacKerell, Computation of the influence of chemical substitution on the $\mathrm{pK}_{a}$ of pyridine using semiempirical and ab initio methods, Theoretical Chemistry Accounts 103 483-494(2000).

[77] F. D'Souza, M.E. Zandler, G.R. Deviprasad, and W. Kutner, Acid-base properties of fulleropyrrolidines: Experimental and theoretical investigations, Journal of Physical Chemistry A 104 6887-6893(2000).

[78] D.E. Elmore and D.A. Dougherty, A computational study of nicotine conformations in the gas phase and in water, Journal of Organic Chemistry 65 742-747(2000).

[79] F. Iribarne, M. Paulino, and O. Tapia, Hydride-transfer transition structure as a possible unifying redox step for describing the branched mechanism of glutathione reductase. Molecular-electronic antecedents, Theoretical Chemistry Accounts 103 451-462(2000).

[80] A. Rastelli, R. Gandolfi, and M.S. Amade, Regioselectivity and diastereoselectivity in the 1,3dipolar cycloadditions of nitrones with acrylonitrile and maleonitrile. The origin of endo/exo selectivity, Advances in Quantum Chemistry 36 151-167(2000).

[81] M.M. Wang, B. Cornett, J. Nettles, D.C. Liotta, and J.P. Snyder, The oxetane ring in taxol, Journal of Organic Chemistry 65 1059-1068(2000).

[82] C.S. Callam, S.J. Singer, T.L. Lowary, and C.M. Hadad, Computational analysis of the potential energy surfaces of glycerol in the gas and aqueous phases: Effects of level of theory, basis set, and solvation on strongly intramolecularly hydrogen-bonded systems, Journal of the American Chemical Society 123 11743-11754(2001).

[83] E. Lodyga-Chruscinska, G. Micera, D. Sanna, J. Olczak, and J. Zabrocki, A new class of peptide chelating agents towards copper(II) ions, Polyhedron 20 1915-1923(2001).

[84] H. Matter, K.H. Baringhaus, T. Naumann, T. Klabunde, and B. Pirard, Computational approaches towards the rational design of drug-like compound libraries, Combinatorial Chemistry \& High Throughput Screening 4 453-475(2001).

[85] G. Tresadern, J. Willis, I.H. Hillier, and C.I.F. Watt, Hydride shift in substituted phenyl glyoxals: Interpretation of experimental rate data using electronic structure and variational transition state theory calculations, Physical Chemistry Chemical Physics 3 3967-3972(2001).

[86] P. Benedetti, R. Mannhold, G. Cruciani, and M. Pastor, GBRcompounds and mepyramines as cocaine abuse therapeutics: Chemometric studies on selectivity using grid independent descriptors (grind), Journal of Medicinal Chemistry 45 1577-1584(2002).

[87] E.J. Delgado and J. Alderete, On the calculation of Henry's law constants of chlorinated benzenes in water from semiempirical quantum chemical methods, Journal of Chemical Information and Computer Science 42 559-563(2002). 
[88] M. Filizola and D.L. Harris, Molecular determinants of recognition and activation at GABA(a)/benzodiazepine receptors, International Journal of Quantum Chemistry 88 5664(2002).

[89] C. Fontanesi, R. Benassi, R. Giovanardi, M. Marcaccio, F. Paolucci, and S. Roffia, Computational electrochemistry. Ab initio calculation of solvent effect in the multiple electroreduction of polypyridinic compounds, Journal of Molecular Structure 612 277286(2002).

[90] B.J. McConkey, V. Sobolev, and M. Edelman, The performance of current methods in ligandprotein docking, Current Science $\mathbf{8 3} 845-856$ (2002).

[91] E.J. Delgado and J.B. Alderete, Prediction of Henry's law constants of triazine derived herbicides from quantum chemical continuum solvation models, Journal of Chemical Information and Computer Sciences 43 1226-1230(2003).

[92] D. Ivanov and M. Constantinescu, Computational study of maleamic acid cyclodehydration, Journal of Physical Organic Chemistry 16 348-354(2003).

[93] J.W. Storer, D.J. Giesen, C.J. Cramer, and D.G. Truhlar, Class IV charge models: A new semiempirical approach in quantum chemistry, Journal of Computer-Aided Molecular Design 9 87-110(1995).

[94] D.J. Giesen, J.W. Storer, C.J. Cramer, and D.G. Truhlar, A general semiempirical quantum mechanical solvation model for nonpolar solvation free energies. $n$-hexadecane, Journal of the American Chemical Society 117 1057-1068(1995).

[95] D.J. Giesen, C.J. Cramer, and D.G. Truhlar, A semiempirical quantum mechanical solvation model for solvation free energies in all alkane solvents, Journal of Physical Chemistry 99 7137-7146(1995).

[96] S.E. Barrows, F.J. Dulles, C.J. Cramer, D.G. Truhlar, and A.D. French, Factors controlling the relative stability of alternative chair forms and hydroxymethyl conformations of D-glucopyranose, Carbohydrate Research 276 219-251(1995).

[97] A. Bondi, van der Waals volumes and radii, Journal of Physical Chemistry 68 441-451(1964).

[98] G.D. Hawkins, C.J. Cramer, and D.G. Truhlar, Parameterized models of aqueous free energies of solvation based on pairwise descreening of solute atomic charges from a dielectric medium, Journal of Physical Chemistry 100 19824-19839(1996).

[99] J. Srinivasan, M.W. Trevathan, P. Beroza, and D.A. Case, Application of a pairwise generalized Born model to proteins and nucleic acids: Inclusion of salt effects, Theoretical Chemistry Accounts 101 426-434(1999).

[100] C.P. Sosa, T. Hewitt, M.R. Lee, and D.A. Case, Vectorization of the generalized Born model for molecular dynamics on shared-memory computers, Journal of Molecular Structure (Theochem) 549 193-201(2001).

[101] C.C. Chambers, G.D. Hawkins, C.J. Cramer, and D.G. Truhlar, Model for aqueous solvation based on class IV atomic charges and first solvation shell effects, Journal of Physical Chemistry 100 16385-16398(1996).

[102] D.J. Giesen, M.Z. Gu, C.J. Cramer, and D.G. Truhlar, A universal organic solvation model, Journal of Organic Chemistry 61 8720-8721 (1996).

[103] D.J. Giesen, G.D. Hawkins, D.A. Liotard, C.J. Cramer, and D.G. Truhlar, A universal model for the quantum mechanical calculation of free energies of solvation in non-aqueous solvents, Theoretical Chemistry Accounts 98 85-109(1997).

[104] M.H. Abraham, Scales of solute hydrogen-bonding: Their construction and application to physicochemical and biochemical processes, Chemical Society Reviews 73-83(1993).

[105] D.J. Giesen, C.C. Chambers, C.J. Cramer, and D.G. Truhlar, Solvation model for chloroform based on class IV atomic charges, Journal of Physical Chemistry B 101 2061-2069(1997).

[106] M.J.S. Dewar and W. Thiel, Ground states of molecules. 38. The MNDO method. Approximations and parameters, Journal of the American Chemical Society 99 48994907(1977).

[107] W. Thiel and A.A. Voityuk, Extension of MNDO to d orbitals: Parameters and results for the halogens, International Journal of Quantum Chemistry 44 807-829(1992).

[108] W. Thiel and A.A. Voityuk, Extension of MNDO to d orbitals: Integral approximations and preliminary numerical results, Theoretica Chimica Acta 81 391-404(1992).

[109] W. Thiel and A.A. Voityuk, Extension of MNDO to d orbitals: Parameters and results for the second-row elements and for the zinc group, Journal of Physical Chemistry 100616 626(1996). 
[110] G.D. Hawkins, C.J. Cramer, and D.G. Truhlar, Parameterized model for aqueous free energies of solvation using geometry-dependent atomic surface tensions with implicit electrostatics, Journal of Physical Chemistry B $\mathbf{1 0 1}$ 7147-7157(1997).

[111] G.D. Hawkins, D.A. Liotard, C.J. Cramer, and D.G. Truhlar, OMNISOL: Fast prediction of free energies of solvation and partition coefficients, Journal of Organic Chemistry 63 43054313(1998).

[112] G.D. Hawkins, B.J. Lynch, C.P. Kelly, D.A. Liotard, C.J. Cramer, and D.G. Truhlar, OMNISOL-version 2.0, University of Minnesota, Minneapolis, 2005.

[113] D.M. Dolney, G.D. Hawkins, P. Winget, D.A. Liotard, C.J. Cramer, and D.G. Truhlar, A universal solvation model based on the conductor-like screening model, Journal of Computational Chemistry 21 340-366(2000).

[114] A. Klamt and G. Schüürmann, COSMO: A new approach to dielectric screening in solvents with explicit expressions for the screening energy and its gradient, Journal of the Chemical Society, Perkin Transactions II 799-805(1993).

[115] J. Li, T. Zhu, C.J. Cramer, and D.G. Truhlar, A new class IV charge model for extracting accurate partial charges from wave functions, Journal of Physical Chemistry A 102 18201831(1998).

[116] J. Li, J. Xing, C.J. Cramer, and D.G. Truhlar, Accurate dipole moments from Hartree-Fock calculations by means of class IV charges, Journal of Chemical Physics 111 885-892(1999).

[117] P.-O. Löwdin, On the non-orthogonality problem connected with the use of atomic wave functions in the theory of molecules and crystals, Journal of Chemical Physics 18365 375(1950).

[118] I. Mayer, Charge, bond order and valence in the ab initio SCF theory, Chemical Physics Letters 97 270-277(1983).

[119] I. Mayer, Comments on the quantum theory of valence and bonding: Choosing between alternative definitions, Chemical Physics Letters 110 440(1984).

[120] J. Li, G.D. Hawkins, C.J. Cramer, and D.G. Truhlar, Universal reaction field model based on ab initio Hartree-Fock theory, Chemical Physics Letters 288 293-298(1998).

[121] R.E. Easton, D.J. Giesen, A. Welch, C.J. Cramer, and D.G. Truhlar, The MIDI! Basis set for quantum mechanical calculations of molecular geometries and partial charges, Theoretica Chimica Acta 93 281-301(1996).

[122] J. Li, C.J. Cramer, and D.G. Truhlar, MIDI! Basis set for silicon, bromine, and iodine, Theoretical Chemistry Accounts 99 192-196(1998).

[123] T. Zhu, J. Li, G.D. Hawkins, C.J. Cramer, and D.G. Truhlar, Density-functional solvation model based on CM2 atomic charges, Journal of Chemical Physics 109 9117-9133(1998).

[124] J. Li, T. Zhu, G.D. Hawkins, P. Winget, D.A. Liotard, C.J. Cramer, and D.G. Truhlar, Extension of the platform of applicability of the SM5.42R universal solvation model, Theoretical Chemistry Accounts 103 9-63(1999).

[125] P. Winget, J.D. Thompson, C.J. Cramer, and D.G. Truhlar, Parameterization of universal solvation model for molecules containing silicon, Journal of Physical Chemistry A 1065160 5168(2002).

[126] J. Li, B. Williams, C.J. Cramer, and D.G. Truhlar, A class IV charge model for molecular excited states, Journal of Chemical Physics 110 724-733(1999).

[127] M.C. Zerner, Semiempirical molecular orbital methods, Reviews in Computational Chemistry, K.B. Lipkowitz and D.B. Boyd (Eds.), VCH, New York, 1991, pp. 313-365.

[128] J.E. Ridley and M.C. Zerner, An intermediate neglect of differential overlap technique for spectroscopy: Pyrrole and the azines, Theoretica Chimica Acta 32 111(1973).

[129] J. Li, T. Zhu, C.J. Cramer, and D.G. Truhlar, A universal solvation model based on class IV charges and the intermediate neglect of differential overlap for spectroscopy molecular orbital method, Journal of Physical Chemistry B 104 2178-2182(2000).

[130] J. Li, C.J. Cramer, and D.G. Truhlar, A two-response-time model based on CM2/INDO/S2 electrostatic potentials for the dielectric polarization component of solvatochromic shifts on vertical excitation energies, International Journal of Quantum Chemistry 77 264-280(2000).

[131] T. Zhu, J. Li, D.A. Liotard, C.J. Cramer, and D.G. Truhlar, Analytical gradients of a selfconsistent reaction-field solvation model based on CM2 atomic charges, Journal of Chemical Physics 110 5503-5513(1999). 
[132] P. Winget, J.D. Thompson, J.D. Xidos, C.J. Cramer, and D.G. Truhlar, Charge Model 3: A class IV charge model based on hybrid density functional theory with variable exchange., Journal of Physical Chemistry A 106 10707-10717(2002).

[133] J.D. Thompson, C.J. Cramer, and D.G. Truhlar, Parameterization of Charge Model 3 for AM1, PM3, BLYP, and B3LYP, Journal of Computational Chemistry 24 1291-1304(2003).

[134] J.M. Brom, B.J. Schmitz, J.D. Thompson, C.J. Cramer, and D.G. Truhlar, A class IV charge model for boron based on hybrid density functional theory, Journal of Physical Chemistry A 107 6483-6488(2003).

[135] J.A. Kalinowski, B. Lesyng, J.D. Thompson, C.J. Cramer, and D.G. Truhlar, Class iv charge model for the self-consistent charge density-functional tight-binding method, Journal of Physical Chemistry A 108 2545-2549(2004).

[136] J.D. Thompson, J.D. Xidos, T.M. Sonbuchner, C.J. Cramer, and D.G. Truhlar, More reliable partial atomic charges when using diffuse basis sets, PhysChemComm 5 117-134(2002).

[137] J.D. Thompson, C.J. Cramer, and D.G. Truhlar, New universal solvation model and comparison of the accuracy of the SM5.42R, SM5.43R, C-PCM, D-PCM, and IEF-PCM continuum solvation models for aqueous and organic solvation free energies and for vapor pressures, Journal of Physical Chemistry A 108 6532-6542(2004).

[138] J.D. Thompson, C.J. Cramer, and D.G. Truhlar, Density-functional theory and hybrid densityfunctional theory continuum solvation models for aqueous and organic solvents: Universal SM5.43 and SM5.43R solvation models for any fraction of Hartree-Fock exchange, Theoretical Chemistry Accounts 113 107-131(2005).

[139] A.D. Becke, Density functional exchange energy approximation with correct asymptotic behavior, Physical Review A 38 3098-3100(1988).

[140] C. Lee, W. Yang, and R.G. Parr, Development of the Colle-Salvetti correlation-energy formula into a functional of the electron density, Physical Review B 37 785-789(1988).

[141] A.D. Becke, Density-functional thermochemistry. III. The role of exact exchange, Journal of Chemical Physics 98 5648-5652(1993).

[142] P.J. Stephens, F.J. Devlin, C.F. Chabalowski, and M.J. Frisch, Ab initio calculation of vibrational absorption and circular dichroism spectra using density functional force fields, Journal of Physical Chemistry 98 11623-11627(1994).

[143] W.J. Hehre, L. Radom, P.v.R. Schleyer, and J.A. Pople, Ab Initio Molecular Orbital Theory, Wiley, New York, 1986.

[144] J. Perdew and Y. Wang, Accurate and simple analytic representation of the electron-gas correlation energy, Physical Review B 45 13244-13249(1992).

[145] J.P. Perdew, Unified theory of exchange and correlation beyond the local density approximation, Electronic structure of solids '91, P. Ziesche and H. Eschrig (Eds.), Akademie Verlag, Berlin, 1991, pp. 11-20.

[146] C. Adamo and V. Barone, Exchange functionals with improved long-range behavior and adiabatic connection methods without adjustable parameters: The $m \mathrm{PW}$ and $m \mathrm{PW} 1 \mathrm{PW}$ models, Journal of Chemical Physics 108 664-675(1998).

[147] J. Pu, C.P. Kelly, J.D. Thompson, J.D. Xidos, J. Li, T. Zhu, G.D. Hawkins, Y.-Y. Chuang, P.L. Fast, B.J. Lynch, D.A. Liotard, D. Rinaldi, J. Gao, C.J. Cramer, and D.G. Truhlar, GAMESSPLUS version 4.7, University of Minnesota, Minneapolis, 2005.

[148] H. Nakamura, A.C. Chamberlin, C.P. Kelly, J.D. Xidos, J.D. Thompson, J. Li, G.D. Hawkins, T. Zhu, B.J. Lynch, Y. Volobuev, D. Rinaldi, D.A. Liotard, C.J. Cramer, and D.G. Truhlar, HONDOPLUS-v.5.0, University of Minnesota, Minneapolis, MN, 2006.

[149] A.C. Chamberlin, C.P. Kelly, J.D. Thompson, B.J. Lynch, J.D. Xidos, G.D. Hawkins, J. Li, T. Zhu, Y. Volobuev, D. Rinaldi, D.A. Liotard, C.J. Cramer, and D.G. Truhlar, SMXGAUSSversion 3.4, University of Minnesota, Minneapolis, 2006.

[150] S. Miertus, E. Scrocco, and J. Tomasi, Electrostatic interaction of a solute with a continuum. A direct utilization of ab initio molecular potentials for the prevision of solvent effects, Chemical Physics 55 117-129(1981).

[151] M. Cossi, V. Barone, R. Cammi, and J. Tomasi, Ab initio study of solvated molecules: A new implementation of the polarizable continuum model, Chemical Physics Letters 255327 335(1996).

[152] E. Cancès, B. Mennucci, and J. Tomasi, A new integral equation formalism for the polarizable continuum model: Theoretical background and applications to isotropic and anisotropic dielectrics, Journal of Chemical Physics 107 3032-3041(1997). 
[153] M. Cossi, V. Barone, B. Mennucci, and J. Tomasi, Ab initio study of ionic solutions by a polarizable continuum dielectric model, Chemical Physics Letters 286 253-260(1998).

[154] V. Barone and M. Cossi, Quantum calculation of molecular energies and energy gradients in solution by a conductor solvent model, Journal of Physical Chemistry A 102 1995-2001(1998).

[155] M. Cossi, N. Rega, G. Scalmani, and V. Barone, Energies, structures, and electronic properties of molecules in solution with the C-PCM solvation model, Journal of Computational Chemistry 24 669-681(2003).

[156] C.P. Kelly, C.J. Cramer, and D.G. Truhlar, SM6: A density functional theory continuum solvation model for calculating aqueous solvation free energies of neutrals, ions, and solutewater clusters, Journal of Chemical Theory and Computation 1 1133-1152(2005).

[157] W.L. Jorgensen, D.S. Maxwell, and J. Tirado-Rives, Development and testing of the OPLS all-atom force field on conformational energetics and properties of organic liquids, Journal of the American Chemical Society 118 11225-11236(1996).

[158] A.C. Chamberlin, C.J. Cramer, and D.G. Truhlar, Predicting aqueous free energies of solvation as functions of temperature, Journal of Physical Chemistry B 110 5665-5675(2006).

[159] P. Winget, G.D. Hawkins, C.J. Cramer, and D.G. Truhlar, Prediction of vapor pressures from self-solvation free energies calculated by the SM5 series of universal solvation models, Journal of Physical Chemistry B 104 4726-4734(2000).

[160] J.D. Thompson, C.J. Cramer, and D.G. Truhlar, Predicting aqueous solubilities from aqueous free energies of solvation and experimental or calculated vapor pressures of pure substances, Journal of Chemical Physics 119 1661-1670(2003).

[161] P. Winget, C.J. Cramer, and D.G. Truhlar, Prediction of soil sorption coefficients using a universal solvation model, Environmental Science and Technology 34 4733-4740(2000).

[162] W.J. Lyman, Adsorption coefficient for soils and sediments, Handbook of Chemical Property Estimation Methods, W.J. Lyman, W.F. Reehl, and D.H. Rosenblatt (Eds.), American Chemical Society, Washington, DC, 1990, pp. Chapter 4.

[163] C.J. Cramer and D.G. Truhlar, Polarization of the nucleic acid bases in aqueous solution, Chemical Physics Letters 198 74-80(1992).

[164] D.J. Giesen, C.C. Chambers, C.J. Cramer, and D.G. Truhlar, What controls partitioning of the nucleic acid bases between chloroform and water? Journal of Physical Chemistry B 1015084 5088(1997).

[165] D.A. McQuarrie, Statistical Mechanics, Harper \& Row, New York, 1976.

[166] C.P. Kelly, C.J. Cramer, and D.G. Truhlar, Predicting adsorption coefficients at air-water interfaces using universal solvation and surface area models, Journal of Physical Chemistry B 108 12882-12897(2004).

[167] Q. Du, R. Superfine, E. Freysz, and Y.R. Shen, Vibrational spectroscopy of water at the vapor/water interface, Physical Review Letters 70 2313(1993).

[168] Q. Du, E. Freysz, and Y.R. Shen, Surface vibrational spectroscopic studies of hydrogenbonding and hydrophobicity, Science (Washington DC) 264 826-828(1994).

[169] D.E. Gragson and G.L. Richmond, Investigations of the structure and hydrogen bonding of water molecules at liquid surfaces by vibrational sum frequency spectroscopy, Journal of Physical Chemistry B 102 3847-3861(1998).

[170] I.-F.W. Kuo and C.J. Mundy, An ab initio molecular dynamics study of the aqueous liquidvapor interface, Science (Washington DC) 303 658-660(2004).

[171] M.H. Abraham, J. Andonian-Haftvan, G.S. Whiting, A. Leo, and R.S. Taft, Hydrogen bonding. Part 34. The factors that influence the solubility of gases and vapours in water at $298 \mathrm{~K}$, and a new method for its determination, Journal of the Chemical Society, Perkin Transactions II 1777-1791(1994).

[172] M.D. Tissandier, K.A. Cowen, W.Y. Feng, E. Gundlach, M.H. Cohen, A.D. Earhart, J.V. Coe, and T.R. Tuttle, The proton's absolute aqueous enthalpy and Gibbs free energy of solvation from cluster-ion solvation data, Journal of Physical Chemistry A 102 7787-7794(1998).

[173] A. Lewis, J.A. Bumpus, D.G. Truhlar, and C.J. Cramer, Molecular modeling of environmentally important processes: Reduction potentials, Journal of Chemical Education $\mathbf{8 1}$ 596-604(2004).

[174] C.P. Kelly, C.J. Cramer, and D.G. Truhlar, Aqueous solvation free energies of ions and ionwater clusters based on an accurate value for the absolute aqueous solvation free energy of the proton, Journal of Physical Chemistry B 110 16066-16081 (2006). 
[175] E.V. Patterson, C.J. Cramer, and D.G. Truhlar, Reductive dechlorination of hexachloroethane in the environment. Mechanistic studies via computational electrochemistry, Journal of the American Chemical Society 123 2025-2031(2001).

[176] C.J. Cramer and S.M. Gustafson, Hyperconjugation vs. apicophilicity in trigonal bipyramidal phosphorus species, Journal of the American Chemical Society 115 9315-9316(1993).

[177] C.P. Kelly, C.J. Cramer, and D.G. Truhlar, Adding explicit solvent molecules to continuum solvent calculations for the calculation of aqueous acid dissociation constants, Journal of Physical Chemistry A 110 2493-2499(2006).

[178] J. Seckute, J.L. Menke, R.J. Emnett, E.V. Patterson, and C.J. Cramer, Ab initio molecular orbital and density functional studies on the solvolysis of sarin and $O, S$ -

dimethylmethylphosphonothioate, a VX-like compound, Journal of Organic Chemistry 70 8649-8660(2005). 